\title{
A class of nonlocal variational problems on a vector bundle for color image local contrast reduction/enhancement*
}

\author{
Thomas Batard and Marcelo Bertalmío
}

\begin{abstract}
We extend two existing variational models from the Euclidean space to a vector bundle over a Riemannian manifold. The Euclidean models, dedicated to regularize or enhance some color image features, are based on the concept of nonlocal gradient operator acting on a function of the Euclidean space. We then extend these models by generalizing this operator to a vector bundle over a Riemannian manifold with the help of the parallel transport map associated to some class of covariant derivatives. Through the dual formulations of the proposed models, we obtain the expressions of their solutions, which exhibit the functional spaces that describe the image features. Finally, for a well-chosen covariant derivative and its nonlocal extension, the proposed models perform local contrast modification (reduction or enhancement) and experiments show that they preserve more the aspect of the original image than the Euclidean models do while modifying equally its contrast.
\end{abstract}

AMS 2000 SubjeCt Classifications: Primary 49M29, 53C05, 68U10; secondary 90C25, 90C26.

KEYWORDS AND PHRASES: Nonlocal variational model, vector bundle, covariant derivative, image contrast reduction/enhancement, primal-dual algorithm.

1 Introduction

\subsection{Variational techniques for image features regular- ization and enhancement}

1.2 Previous work and contribution

1.3 Connection with human vision models

*This work was supported by the European Research Council, Starting Grant ref. 306337, by the Spanish government, grant ref. TIN2012-38112, and by the Icrea Academia Award. 
2 Nonlocal total variation on a vector bundle

2.1 Nonlocal vectorial total variation on an Euclidean space

2.2 Nonlocal covariant derivative

2.3 Nonlocal total variation on a vector bundle

3 Variational models for image processing tasks

3.1 Image features regularization 201

$\begin{array}{lll}3.1 .1 & \text { Previous work } & 201\end{array}$

3.1.2 The proposed model and its solutions 202

3.1.3 Algorithm to solving the proposed model 203

3.2 Image features enhancement 204

$\begin{array}{lll}3.2 .1 & \text { Previous work } & 204\end{array}$

3.2.2 The proposed model and its solutions 205

3.2.3 Algorithm to solving the proposed model 207

4 Applications to local contrast modification 208

4.1 The chosen nonlocal covariant derivative and its numerical implementation 208

4.1.1 The chosen local covariant derivative 208

4.1.2 Nonlocal extension of the chosen covariant derivative

4.2 Numerical implementation of the algorithms, its limits and solutions to overcome them

$\begin{array}{lll}4.3 & \text { Local contrast reduction } & 215\end{array}$

$\begin{array}{lll}4.4 & \text { Local contrast enhancement } & 218\end{array}$

4.5 Functional spaces modeling noise, textures and contrast on color images

4.5.1 On the relation between large window sizes and contrast 


\subsubsection{On the relation between small window sizes and noise/textures}

4.5.3 On the residual term

5 Conclusion and further work

232

Acknowledgements

233

References

233

\section{Introduction}

\subsection{Variational techniques for image features regularization and enhancement}

Regularizing or enhancing image features, e.g. noise, textures, edges, contrast, are very useful tasks for correcting defects produced during the acquisition process of a real-world scene and its reproduction on a display, due to physical and technological limitations (see e.g. [6] for more details). These tasks are also useful as pre-processing stage in order to improve the results of higher level applications like pattern recognition. Variational methods are then a very powerful tool for performing regularization or enhancement of image features, and have been extensively used over the last two decades.

Regarding enhancement tasks, a seminal variational approach is due to Sapiro and Caselles [34] for contrast enhancement purpose. Later on, Bertalmío et al. [7] adapted that model to deal with local contrast, and several applications have then been derived from that variational model: perceptual color correction [7],[30], tone mapping [15], color gamut expansion [41], and dehazing [18] to name a few. Connections have also been made between the variational formulation [7] and Retinex theory [8],[9]. As enhancing some features can also yield noise amplification, some techniques combine features enhancement with noise removal (see e.g. [40] for coherence enhancement on color images, [17] for edge enhancement on gray-level images, [2] for color enhancement. Note that these three methods are based on generalized heat diffusions, and do not necessarily arise from a variational formulation. More recently, Fitschen et al. [16] proposed a variational model for contrast enhancement on color images with the total variation (TV) as regularizing term.

The Rudin-Osher-Fatemi (ROF) denoising model [33] based on minimizing the TV of the image has been a pioneering approach for removing noise 
from images. However, as it has also a tendency to smooth textures and reduce the contrast of the image, several modifications of the TV have then been proposed in order to tackle those issues (see e.g. [10],[42] for second order derivatives-based regularizers and [4],[11],[21] for vectorial extensions of the TV). On the other hand, since the seminal nonlocal approaches of Buades et al. [12] and Awate et al. [1] for image denoising, several nonlocal variational formulations for image regularization have been proposed (see e.g. [20],[23],[25]). In particular, Gilboa and Osher [20] introduced the concept of nonlocal gradient operator from which they derive a nonlocal total variation $\left(\mathrm{TV}_{w}^{\mathrm{NL}}\right)$, associated to some weight function $w$. Whereas the aforementioned models in this paragraph are devoted to noise or irregularities removal, the contrast enhancement model of Bertalmío et al. [7] can be slightly modified to produce contrast reduction [8], which has been used by Zamir et al. [41] for color gamut reduction.

\subsection{Previous work and contribution}

In [4], we extended the ROF denoising model (based on TV) and its vectorial extension proposed by Bresson and Chan [11] (based on a vectorial extension of TV, called VTV) to a vector bundle over a Riemannian manifold, replacing the Euclidean gradient operator in the expression of TV and the Jacobian operator in the expression of VTV by a covariant derivative. In particular, we showed that, if the covariant derivative is compatible with the vector bundle metric, then the corresponding total variation VBTV possesses a dual formulation from which follows an expression of the unique solution of the variational model. We also derived from the dual formulation of the variational problem an algorithm to compute the numerical solution, based on Chambolle's projection algorithm [13]. For a well-chosen covariant derivative, we showed that our model preserves better the edges and textures of the original image than the TV-based model for gray-level images and the VTV-based model for color images. Moreover, it also outperforms these "Euclidean" models in terms of objective measures, namely the Peak Signal-to-Noise Ratio (PSNR) and the image Quality index (Q-index) [37], this latter being more correlated to perception than the PSNR [31].

Based on the observation that the nonlocal regularization model of Gilboa and Osher [20] associated to the so-called anisotropic $\mathrm{TV}_{w}^{\mathrm{NL}}$ and the contrast enhancement model of Bertalmío et al. [7] are similar up to the sign of the anisotropic $\mathrm{TV}_{w}^{\mathrm{NL}}$ in their variational formulations, we established in [5] some connections between the solutions of these models. Under their dual formulations, we showed that the expressions of the solutions both exhibit 
the same functional space, which is a nonlocal extension of the space of oscillating patterns [29] that is involved in the expression of the solution of the ROF model. We then showed that Chambolle's projection algorithm can be extended to the nonlocal case to solve the (convex) regularization model and can be adapted to deal with the (non convex) enhancement model and approximate a solution. Note that we showed that these results also hold in the vectorial setting, i.e. when considering a nonlocal vectorial total variation $\mathrm{VTV}_{w}^{\mathrm{NL}}$.

This paper can be viewed as an unification of our two previous works aforementioned: we extend the nonlocal models studied in [5] from the Euclidean space to a vector bundle over a Riemannian manifold equipped with a vector bundle metric, as we did in the local case in [4]. For this, we introduce the concept of nonlocal covariant derivative on a vector bundle, by the use of the parallel transport map associated to a covariant derivative. We then construct a nonlocal total variation $\mathrm{VBTV}_{w}^{\mathrm{NL}}$ induced by the nonlocal covariant derivative and we show that it possesses a dual formulation provided that the (local) covariant derivative is compatible with the vector bundle metric. By the use of the dual formulations of the corresponding variational models, we obtain expressions of their solutions, and we show that they can be computed numerically through primal-dual algorithms (see e.g. [14]), which are known to be more efficient than the projection algorithm we were using in [5]. The expressions of the solutions also exhibit a class of functional spaces, which can be viewed as nonlocal and non Euclidean extensions of the space of oscillating patterns aforementioned. Finally, taking the covariant derivative compatible with the vector bundle metric constructed in [3] and $w$ as a Gaussian kernel, experiments show that these functional spaces can model noise, textures and contrast on color images, and the variational models perform local contrast modification (reduction or enhancement) that preserves more the aspect of the original image than their "Euclidean" restrictions in [5] while modifying equally its contrast.

\subsection{Connection with human vision models}

As mentioned earlier in the Introduction, the contrast enhancement model presented in this paper is a two-folds extension of the model of Bertalmío et al. [7]: first, we extended this channel-wise model in a vectorial Euclidean way in [5], that we extend to a vector bundle in this paper. The model of Bertalmío et al. has been designed in order to reproduce some properties of the Human Visual System (HSV) like local contrast enhancement, visual adaptation, and color constancy. This model is closely related to the original 
Retinex theory of Land and McCann [26] whose aim was to reproduce the sensory response to color stimuli by the HSV.

Georgiev [19] pointed out some limitations of Retinex original formulation, and proposed a mathematical model of relighting and adaptation of human vision based on von Kries model [39]. In Georgiev's model, a color image is considered as a section of a vector bundle and the perceived difference of brightness between two neighboring pixels is encoded by a covariant derivative.

Theoretically, the model we are presenting in this paper can then be viewed a combination of the Retinex-based model of Bertalmío et al. and the one of Georgiev. This suggests that the model we are proposing may encode new properties of the HSV.

\section{Nonlocal total variation on a vector bundle}

\subsection{Nonlocal vectorial total variation on an Euclidean space}

The aim of this section is to remind the reader of the definition of vectorial nonlocal total variation $\mathrm{VTV}_{w}^{\mathrm{NL}}$ we introduced in [5].

Definition 2.1 (Nonlocal gradient operator). Let $\Omega$ be a compact subset of $\mathbb{R}^{2}$ and $u: \Omega \longrightarrow \mathbb{R}^{n}$ be a smooth vector-valued function. We assume that $\mathbb{R}^{n}$ is equipped with a positive definite quadratic form $h$. A nonlocal gradient is an operator $\nabla_{w}^{N L}: C^{\infty}\left(\Omega ; \mathbb{R}^{n}\right) \longrightarrow C^{\infty}\left(\Omega \times \Omega ; \mathbb{R}^{n}\right)$ of the form

$$
\nabla_{w}^{N L} u:(x, y) \longmapsto w(x, y)(u(y)-u(x))
$$

and $w: \Omega \times \Omega \longrightarrow \mathbb{R}^{+*}$ is a smooth symmetric function.

Note that formula (1) is nothing but the vectorial extension of the nonlocal gradient introduced by Gilboa and Osher [20] for real-valued functions.

Standard choices for the weight function $w$ in the context of image processing are Euclidean/Riemannian distance, Gaussian kernel, and patchbased distances. We refer to Zosso et al. [38] for more details as well as for the definition of a weight function specific to color images determined by the hue difference between two colors. The choice of the positive definite quadratic form $h$ greatly depends on the nature of the image to be processed. Dealing with color images, a suitable choice for $h$ is the perceptual metric associated to the color space involved (e.g. the Euclidean metric associated to the CIE Lab color space).

Definition 2.2 (The space $W_{w, 1, p}^{N L}\left(\Omega ; \mathbb{R}^{n}\right)$ ). The quadratic form $h$ induces a scalar product $\langle$,$\rangle on C^{\infty}\left(\Omega \times \Omega ; \mathbb{R}^{n}\right)$ defined by 


$$
\left\langle\eta_{1}, \eta_{2}\right\rangle:=\int_{\Omega \times \Omega}\left(\eta_{1}(x, y), \eta_{2}(x, y)\right)_{h} d x d y
$$

where $(,)_{h}$ denotes the scalar product with respect to $h$.

The $L^{p}$ norm on $C^{\infty}\left(\Omega \times \Omega ; \mathbb{R}^{n}\right)$ is defined by

$$
\|\eta\|_{L^{p}}:=\left(\int_{\Omega \times \Omega}\|\eta(x, y)\|_{h}^{p} d x d y\right)^{1 / p}
$$

where \|\|$_{h}$ denotes the norm associated to $h$. In particular, we have

$$
\left\|\nabla_{w}^{N L} u\right\|_{L^{p}}=\left(\int_{\Omega \times \Omega}\|w(x, y)(u(y)-u(x))\|_{h}^{p} d x d y\right)^{1 / p} .
$$

Finally, we define the space

$$
W_{w, 1, p}^{N L}\left(\Omega ; \mathbb{R}^{n}\right):=\left\{u \in L^{p}\left(\Omega ; \mathbb{R}^{n}\right): \nabla_{w}^{N L} u \in L^{p}\left(\Omega \times \Omega ; \mathbb{R}^{n}\right)\right\} .
$$

Assuming that the function $u$ is real-valued, the norm $\left\|\nabla_{w}^{N L} u\right\|_{L^{1}}$ corresponds to the anisotropic $\mathrm{TV}_{w}^{\mathrm{NL}}$ introduced by Gilboa and Osher [20], as well as the contrast modification term in the perceptual color correction model proposed by Bertalmío et al. [7].

Definition 2.3 (Adjoint of a nonlocal gradient operator). We define the adjoint of the operator $\nabla_{w}^{N L}$ as the operator $\nabla_{w}^{N L^{*}}: C^{\infty}\left(\Omega \times \Omega ; \mathbb{R}^{n}\right) \longrightarrow$ $C^{\infty}\left(\Omega ; \mathbb{R}^{n}\right)$ satisfying

$$
\left\langle\nabla_{w}^{N L} u, \eta\right\rangle=\left(u, \nabla_{w}^{N L^{*}} \eta\right)
$$

$\forall u \in C^{\infty}\left(\Omega ; \mathbb{R}^{n}\right), \forall \eta \in C^{\infty}\left(\Omega \times \Omega ; \mathbb{R}^{n}\right)$, where $($,$) is the L^{2}$ scalar product on $C^{\infty}\left(\Omega ; \mathbb{R}^{n}\right)$ induced by $h$.

As in the scalar case in [20], a straightforward computation yields

$$
\nabla_{w}^{N L^{*}} \eta: x \longmapsto \int_{\Omega} w(x, y)(\eta(y, x)-\eta(x, y)) d y .
$$

We derive from Def. 2.3 the definition of $\operatorname{VTV}_{w}^{\mathrm{NL}}$ on the set $L^{1}\left(\Omega ; \mathbb{R}^{n}\right)$.

Definition 2.4 (Nonlocal vectorial total variation). The nonlocal vectorial total variation $V T V_{w}^{N L}(u)$ of $u \in L^{1}\left(\Omega ; \mathbb{R}^{n}\right)$ is the quantity

$$
\sup _{\eta \in \mathcal{H}_{1}}\left(\int_{\Omega}\left(u, \nabla_{w}^{N L^{*}} \eta\right)_{h} d \Omega\right)
$$


where the set $\mathcal{H}_{a}$, for $a \in \mathbb{R}^{+*}$, is

$$
\mathcal{H}_{a}:=\left\{\eta \in C^{\infty}\left(\Omega \times \Omega ; \mathbb{R}^{n}\right),\|\eta(x, y)\|_{h} \leq a \forall x, y \in \Omega\right\} .
$$

Note that we can also write

$$
V T V_{w}^{N L}(u)=\sup _{\xi \in K_{1}}\left(\int_{\Omega}(u, \xi)_{h} d \Omega\right),
$$

where the set $K_{a}$ is the closure in $L^{2}\left(\Omega ; \mathbb{R}^{n}\right)$ of the set $\mathcal{K}_{a}$ defined by

$$
\mathcal{K}_{a}:=\left\{\nabla_{w}^{N L^{*}} \eta: \eta \in C^{\infty}\left(\Omega \times \Omega ; \mathbb{R}^{n}\right),\|\eta(x, y)\|_{h} \leq a \forall x, y \in \Omega\right\} .
$$

We denote by $B V_{w}^{N L}\left(\Omega ; \mathbb{R}^{n}\right)$ the set of functions $u \in L^{1}\left(\Omega ; \mathbb{R}^{n}\right)$ such that $V T V_{w}^{N L}(u)<+\infty$.

Proposition 2.1. If $u \in W_{w, 1,1}^{N L}\left(\Omega ; \mathbb{R}^{n}\right)$ then,

$$
V T V_{w}^{N L}(u)=\left\|\nabla_{w}^{N L} u\right\|_{L^{1}}
$$

Proof. Prop. 2.1 is a particular case of Prop. 2.3 whose proof is detailed below.

\subsection{Nonlocal covariant derivative}

In order to extend $\mathrm{VTV}_{w}^{\mathrm{NL}}$ from the Euclidean space to a vector bundle over a Riemannian manifold, we need first to extend the concept of nonlocal gradient operator to a vector bundle. Our proposal is then to make use of the parallel transport map associated to a covariant derivative. We first remind the definitions of these two objects. We refer the reader to [24],[28] for an introduction to Riemannian geometry and vector bundles.

Let $E$ be a vector bundle over a complete Riemannian manifold $(M, g)$ and $\pi: E \longrightarrow M$ the projection map. In this paper, we denote by $E_{x}$ the fiber over $x \in M$, i.e. the set $\left\{\pi^{-1}(x)\right\}$, and by $\Gamma(E)$ the set of smooth sections of $E$. We denote by $T M$ resp. $T^{*} M$ the tangent bundle resp. the cotangent bundle of $M$, and by $\bigwedge^{k} T^{*} M$ the vector bundle of differential $k$ forms of $M$. We denote by $\operatorname{End}(E)$ the bundle of fiber-wise endomorphisms of $E$. Finally, we denote by $\operatorname{pr}_{1}(E)$ the vector bundle over $M \times M$ induced by the projection

$$
\begin{aligned}
M \times M & \longrightarrow M \\
p r_{1}: \quad(x, y) & \longmapsto x
\end{aligned}
$$

In others words 


$$
\operatorname{pr}_{1}(E)=\{(x, y, p) \in M \times M \times E / x=\pi(p)\}
$$

Definition 2.5 (Covariant derivative). A covariant derivative (or connection) on $E$ is a map $\nabla: \Gamma(T M) \times \Gamma(E) \longrightarrow \Gamma(E)$ of the form

$$
\nabla_{X} \varphi=d_{X} \varphi+\omega(X) \varphi
$$

where $X \in \Gamma(T M)$, d stands for the differential operator acting on the components of the sections and $\omega \in \Gamma\left(T^{*} M \otimes E n d(E)\right)$ is called a connection 1-form.

Hence, a covariant derivative is completely determined by a connection 1 -form. The covariant derivative induced by the connection 1 -form $\omega \equiv 0$ is called trivial covariant derivative.

Definition 2.6 (Parallel transport). Let $\gamma: I \subset \mathbb{R} \longrightarrow M$ be a smooth curve. The parallel transport associated to a covariant derivative $\nabla$ on $E$ along the curve $\gamma$ is the map $\tau_{\gamma, t_{1}, t_{2}}: E_{\gamma\left(t_{1}\right)} \longrightarrow E_{\gamma\left(t_{2}\right)}$ such that $\tau_{\gamma, t_{1}, t_{2}}\left(\varphi_{0}\right)$ is the solution of the differential equation

$$
\left\{\begin{aligned}
\nabla_{\gamma^{\prime}(t)} \varphi \circ \gamma(t) & =0 \quad \forall t \in\left[t_{1}, t_{2}\right] \\
\varphi \circ \gamma\left(t_{1}\right) & =\varphi_{0}
\end{aligned}\right.
$$

We have now available the tools to introduce the concept of nonlocal covariant derivative on a vector bundle. We distinguish two cases, whether the covariant derivative is flat or not.

Definition 2.7 (Flat covariant derivative). The curvature associated to a covariant derivative $\nabla$ is the tensor $R \in \Gamma\left(\bigwedge^{2} T^{*} M \otimes\right.$ End $\left.E\right)$ defined by

$$
R(X, Y) \varphi=\nabla_{X} \nabla_{Y} \varphi-\nabla_{Y} \nabla_{X} \varphi-\nabla_{[X, Y]} \varphi
$$

where $X, Y \in \Gamma(T M)$ and $\varphi \in \Gamma(E)$.

The covariant derivative $\nabla$ is said flat if its curvature tensor vanishes identically.

Then, based on the fact that the parallel transport associated to a flat covariant derivative between two points is independent of the curve joining these two points, we propose the following definition.

Definition 2.8 (nonlocal covariant derivative: the case of flat covariant derivative). The nonlocal covariant derivative associated to a flat covariant derivative $\nabla$ is an operator $\nabla_{w}^{N L}: \Gamma(E) \longrightarrow \Gamma\left(p r_{1}(E)\right)$ of the form

$$
\nabla_{w}^{N L} \varphi:(x, y) \longmapsto w(x, y)\left(\tau_{\gamma_{(x, y)}, 0, l\left(\gamma_{(x, y)}\right)}^{-1} \varphi(y)-\varphi(x)\right)
$$


for any smooth curve $\gamma_{(x, y)}$ starting at $x$ and ending at $y$, where $l\left(\gamma_{(x, y)}\right)$ denotes its length, and where $w$ is a symmetric positive function.

As the map $\tau_{\gamma_{(x, y)}, 0, l\left(\gamma_{(x, y)}\right)}^{-1}$ only depends on $x$ and $y$, we rewrite (13) as

$$
\nabla_{w}^{N L} \varphi:(x, y) \longmapsto w(x, y)\left(\tau_{x, y}^{-1} \varphi(y)-\varphi(x)\right)
$$

We can then observe that formulae (13) and (14) restrict to the "Euclidean" nonlocal gradient operator (1) when $\nabla$ is taken as the trivial covariant derivative.

In order to adapt formula (13) to non flat covariant derivatives, we need to specify the curves along which we perform the parallel transport. A natural choice is then to consider the geodesics of $(M, g)$, and this yields the following definition.

Definition 2.9 (nonlocal covariant derivative: general case). The nonlocal covariant derivative associated to a covariant derivative $\nabla$ is an operator $\nabla_{w}^{N L}: \Gamma(E) \longrightarrow \Gamma\left(p r_{1}(E)\right)$ of the form

$$
\nabla_{w}^{N L} \varphi:(x, y) \longmapsto w(x, y)\left(\frac{1}{\sharp\left\{\gamma_{(x, y)}^{\min }\right\}} \sum_{\left\{\gamma_{(x, y)}^{\min }\right\}} \tau_{\gamma_{(x, y)}^{\min }, 0, d_{g}(x, y)}^{-1} \varphi(y)-\varphi(x)\right)
$$

where $\left\{\gamma_{(x, y)}^{\min }\right\}$ is the set of geodesics starting at $x$ and ending at $y$ parametrized by the arc length, $d_{g}(x, y)$ the geodesic distance between $x$ and $y$, i.e. the length of the curves $\gamma_{(x, y)}^{\text {min }}$, and where $w$ is a symmetric positive function.

Both definitions are indeed compatible since formula (15) does reduce to $(13)$ if $\nabla$ is flat.

\subsection{Nonlocal total variation on a vector bundle}

In this section, we introduce the concept of nonlocal total variation $\operatorname{VBTV}_{w}^{\mathrm{NL}}$ for integrable sections of a vector bundle equipped with a definite positive metric and a covariant derivative compatible with that metric. We first remind the reader of some definitions and results related to covariant derivatives compatible with a vector bundle metric.

Definition 2.10. A metric $h$ on a vector bundle $E$ over a manifold $M$ is the assignment of a scalar product $h_{x}$ on each fiber $\left\{\pi^{-1}(x)\right\}, x \in M$. The metric is said positive definite if the scalar products are positive definite.

Definition 2.11. A covariant derivative $\nabla$ is compatible with the vector bundle metric $h$ if it satisfies 


$$
d h(\varphi, \psi)=(\nabla \varphi, \psi)_{h}+(\varphi, \nabla \psi)_{h}
$$

for any $\varphi, \psi \in \Gamma(E)$.

It follows from Def. 2.11 that the parallel transport $\tau_{\gamma, t, s}$ along any smooth curve $\gamma$ associated to a covariant derivative compatible with a metric $h$ is an isometry $\left(E_{\gamma(t)}, h_{\gamma(t)}\right) \longrightarrow\left(E_{\gamma(s)}, h_{\gamma(s)}\right)$.

Moreover, there is a one-one correspondence between connection 1-forms $\omega$ that are $\mathfrak{s o}(h)$-valued i.e. $\omega \in \Gamma\left(T^{*} M \otimes \mathfrak{s o}(h)\right)$ and covariant derivatives $\nabla$ that are compatible with the metric $h$. We refer to Lawson and Michelson ([27], Prop. 4.4 p.103) for a proof of that result.

From now on, we assume that $E$ is equipped with a positive definite metric $h$, and $\nabla$ is a connection compatible with $h$. Under this latter assumption, the nonlocal covariant derivative (15) possesses an adjoint operator, that we will define below. We first introduce a norm on the space $\Gamma\left(p r_{1}(E)\right)$, defined in formula (9).

The positive definite metric $h$ on $E$ induces a $L^{2}$ scalar product $\langle$,$\rangle on$ $\Gamma\left(p r_{1}(E)\right)$ defined by

$$
\left\langle\eta_{1}, \eta_{2}\right\rangle:=\int_{M \times M}\left(\eta_{1}(x, y), \eta_{2}(x, y)\right)_{h_{x}} d x d y
$$

where $(,)_{h_{x}}$ denotes the scalar product in $E_{x}$ with respect to $h(x)$, from which derive the $L^{p}$ norm on $\Gamma\left(p r_{1}(E)\right)$ defined by

$$
\|\eta\|_{L^{p}}:=\left(\int_{M \times M}\|\eta(x, y)\|_{h_{x}}^{p} d x d y\right)^{1 / p}
$$

where \|\|$_{h_{x}}$ denotes the norm associated to $h(x)$, and the space $L^{p}\left(\operatorname{pr}_{1}(E)\right)$ as the completion of $\Gamma\left(p r_{1}(E)\right)$ in this norm.

Finally, we define the space

$$
W_{w, 1, p}^{N L}:=\left\{\varphi \in L^{p}(E), \nabla_{w}^{N L} \varphi \in L^{p}\left(p r_{1}(E)\right)\right\}
$$

Definition 2.12 (Adjoint of nonlocal covariant derivative). The adjoint of a nonlocal covariant derivative $\nabla_{w}^{N L}$ induced by a covariant derivative $\nabla$ compatible with a positive definite metric $h$ is the operator $\nabla_{w}^{N L^{*}}: \Gamma\left(p r_{1}(E)\right) \longrightarrow$ $\Gamma(E)$ satisfying

$$
\left\langle\nabla_{w}^{N L} \varphi, \eta\right\rangle=\left(\varphi, \nabla_{w}^{N L^{*}} \eta\right)
$$

$\forall \varphi \in \Gamma(E), \forall \eta \in \Gamma\left(\operatorname{pr}_{1}(E)\right)$, where $($,$) is the L^{2}$ scalar product on $\Gamma(E)$ induced by $h$.

Proposition 2.2. The operator $\nabla_{w}^{N L^{*}}$ is defined by 
(17)

$$
\nabla_{w}^{N L^{*}} \eta: x \longrightarrow \int_{M} w(x, y)\left(\frac{1}{\sharp\left\{\gamma_{(x, y)}^{\min }\right\}} \sum_{\left\{\gamma_{(x, y)}^{\min }\right\}} \tau_{\gamma_{(x, y)}^{\min }, 0, d_{g}(x, y)}^{-1} \eta(y, x)-\eta(x, y)\right) d y
$$

Proof. We have

$$
\begin{aligned}
\left\langle\nabla_{w}^{N L} \varphi, \eta\right\rangle & \int_{M \times M} w(x, y)\left[\frac { 1 } { \sharp \{ \gamma _ { ( x , y ) } ^ { \operatorname { m i n } } \} } \sum _ { \{ \gamma _ { ( x , y ) } ^ { \operatorname { m i n } } \} } \left(\left(\tau_{\gamma_{(x, y)}^{\min }, 0, d_{g}(x, y)}^{-1} \varphi(y)\right.\right.\right. \\
& \left.-\varphi(x)), \eta(x, y))_{h_{x}}\right] d x d y \\
= & \int_{M \times M} w(x, y)\left[\frac { 1 } { \sharp \{ \gamma _ { ( x , y ) } ^ { \operatorname { m i n } } \} } \sum _ { \{ \gamma _ { ( x , y ) } ^ { \operatorname { m i n } } \} } \left(\left(\varphi(y), \tau_{\gamma_{(x, y)}^{\min }, 0, d_{g}(x, y)} \eta(x, y)\right)_{h_{y}}\right.\right. \\
& \left.\left.-(\varphi(x), \eta(x, y))_{h_{x}}\right)\right] d x d y
\end{aligned}
$$

since $\tau_{\gamma_{(x, y)}^{\min }, 0, d_{g}(x, y)}:\left(E_{x}, h_{x}\right) \longrightarrow\left(E_{y}, h_{y}\right)$ is an isometry, by compatibility of $\nabla$ with $h$.

It then follows

$$
\begin{aligned}
= & \int_{M \times M} w(x, y)\left[\frac { 1 } { \sharp \{ \gamma _ { ( x , y ) } ^ { \operatorname { m i n } } \} } \sum _ { \{ \gamma _ { ( y , x ) } ^ { \operatorname { m i n } } \} } \left(\left(\varphi(x), \tau_{\gamma_{(y, x)}^{\min }, 0, d_{g}(x, y)} \eta(y, x)\right)_{h_{x}}\right.\right. \\
& \left.\left.-(\varphi(x), \eta(x, y))_{h_{x}}\right)\right] d x d y
\end{aligned}
$$

by interchanging $x$ and $y$ in the first part of the integral, and using the symmetry of $w$ and $d_{g}$.

Rearranging the terms and using $\tau_{\gamma_{(y, x)}^{\min }, 0, d_{g}(x, y)}=\tau_{\gamma_{(x, y)}^{\min }, 0, d_{g}(x, y)}^{-1}$, we finally obtain

$$
\begin{aligned}
\left\langle\nabla_{w}^{N L} \varphi, \eta\right\rangle= & \int_{M \times M}(\varphi(x), w(x, y) \\
& \left.\times\left[\frac{1}{\sharp\left\{\gamma_{(x, y)}^{\min \}}\right.} \sum_{\left\{\gamma_{(x, y)}^{\min }\right\}} \tau_{\gamma_{(x, y)}^{\min }, 0, d_{g}(x, y)}^{-1} \eta(y, x)-\eta(x, y)\right]\right)_{h_{x}} d x d y
\end{aligned}
$$


from which we deduce (17).

Definition 2.13 (Nonlocal vectorial total variation of integrable sections). Let $\varphi \in L^{1}(E)$ and $w: M \times M \longrightarrow \mathbb{R}^{+*}$ be a smooth symmetric function, we define the nonlocal vectorial total variation $V B T V_{w}^{N L}(\varphi)$ of $\varphi$ as the quantity

$$
\sup _{\eta \in \mathcal{H}_{1}}\left(\int_{M}\left(\varphi, \nabla_{w}^{N L^{*}} \eta\right)_{h} d M\right)
$$

where the set $\mathcal{H}_{a}$, for $a \in \mathbb{R}^{+*}$, is

$$
\mathcal{H}_{a}:=\left\{\eta \in \Gamma\left(p r_{1}(E)\right),\|\eta(x, y)\|_{h_{x}} \leq a \forall x, y \in M\right\}
$$

We can also write

$$
V B T V_{w}^{N L}(\varphi)=\sup _{\xi \in K_{1}}\left(\int_{M}(\varphi, \xi)_{h} d M\right)
$$

where $K_{a}$ is the closure in $L^{2}(E)$ of the set $\mathcal{K}_{a}$ defined by

$$
\mathcal{K}_{a}:=\left\{\nabla_{w}^{N L^{*}} \eta: \eta \in \Gamma\left(p r_{1}(E)\right),\|\eta(x, y)\|_{h_{x}} \leq a \forall x, y \in M\right\}
$$

We denote by $B V_{w}^{N L}(E)$ the set of sections $\varphi \in L^{1}(E)$ such that $V B T V_{w}^{N L}(\varphi)<+\infty$.

Proposition 2.3. If $\varphi \in W_{w, 1,1}^{N L}(E)$ then,

$$
V B T V_{w}^{N L}(\varphi)=\left\|\nabla_{w}^{N L} \varphi\right\|_{L^{1}}
$$

Proof. Let $\eta \in \Gamma\left(p r_{1}(E)\right)$, we have

$$
\int_{M}\left(\varphi, \nabla_{w}^{N L^{*}} \eta\right)_{h} d M=\int_{M \times M}\left\langle\nabla_{w}^{N L} \varphi, \eta\right\rangle_{h} d M \times M
$$

by definition of the adjoint operator $\nabla_{w}^{N L^{*}}$.

Then, as the metric $h$ is positive definite, we have

$$
\int_{M \times M}\left\langle\nabla_{w}^{N L} \varphi, \eta\right\rangle_{h} d M \times M \leq \int_{M \times M}\left\|\nabla_{w}^{N L} \varphi\right\|_{h}\|\eta\|_{h} d M \times M
$$

and it follows

$$
\int_{M \times M}\left\|\nabla_{w}^{N L} \varphi\right\|_{h}\|\eta\|_{h} d M \times M \leq \int_{M \times M}\left\|\nabla_{w}^{N L} \varphi\right\|_{h} d M \times M
$$


since $\|\eta(x, y)\|_{h_{x}} \leq 1 \quad \forall(x, y) \in M \times M$. Hence

$$
\sup _{\eta \in \mathcal{H}_{1}}\left(\int_{M}\left(\varphi, \nabla_{w}^{N L^{*}} \eta\right)_{h} d M\right) \leq \int_{M \times M}\left\|\nabla_{w}^{N L} \varphi\right\|_{h} d M \times M
$$

Let

$$
\tilde{\eta}:(x, y) \longmapsto\left\{\begin{array}{cl}
\frac{\nabla_{w}^{N L} \varphi(x, y)}{\left\|\nabla_{w}^{N L} \varphi(x, y)\right\|_{h_{x}}} & \text { if } \nabla_{w}^{N L} \varphi(x, y) \neq 0 \\
0 & \text { otherwise }
\end{array}\right.
$$

and $\left(\eta_{\epsilon}\right) \in \Gamma\left(p r_{1}(E)\right),\left\|\eta_{\epsilon}(x, y)\right\|_{h_{x}} \leq 1 \forall(x, y) \in M \times M$ such that $\eta_{\epsilon} \underset{\epsilon \rightarrow 0}{\longrightarrow} \tilde{\eta}$. We claim that such a sequence can be constructed using generalizations of Lusin's theorem and the mollification technique to vector-valued measurable functions.

We then have

$$
\begin{aligned}
\lim _{\epsilon \rightarrow 0} \int_{M}\left(\varphi, \nabla_{w}^{N L^{*}} \eta_{\epsilon}\right)_{h} d M & =\lim _{\epsilon \rightarrow 0} \int_{M \times M}\left\langle\nabla_{w}^{N L} \varphi, \eta_{\epsilon}\right\rangle_{h} d M \times M \\
& =\int_{M \times M}\left\langle\nabla_{w}^{N L} \varphi, \tilde{\eta}\right\rangle_{h} d M \times M \\
& =\int_{\left\{\nabla_{w}^{N L} \varphi \neq 0\right\}}\left\langle\nabla_{w}^{N L} \varphi, \frac{\nabla_{w}^{N L} \varphi}{\left\|\nabla_{w}^{N L} \varphi\right\|_{h}}\right\rangle_{h} d M \times M \\
& =\int_{M \times M}\left\|\nabla_{w}^{N L} \varphi\right\|_{h} d M \times M
\end{aligned}
$$

Hence, we have constructed a sequence $\int_{M}\left(\varphi, \nabla_{w}^{N L^{*}} \eta_{\epsilon}\right)_{h} d M$ converging towards $\int_{M \times M}\left\|\nabla_{w}^{N L} \varphi\right\|_{h} d M \times M$, and such that

$$
\int_{M}\left(\varphi, \nabla_{w}^{N L^{*}} \eta_{\epsilon}\right)_{h} d M \leq \int_{M \times M}\left\|\nabla_{w}^{N L} \varphi\right\|_{h} d M \times M \quad \forall \epsilon
$$

since $\left\|\eta_{\epsilon}(x, y)\right\|_{h_{x}} \leq 1 \quad \forall(x, y) \in M \times M$. Together with (23), it shows that

$$
\sup _{\eta \in \mathcal{H}_{1}}\left(\int_{M}\left(\varphi, \nabla_{w}^{N L^{*}} \eta\right)_{h} d M\right)=\int_{M \times M}\left\|\nabla_{w}^{N L} \varphi\right\|_{h} d M \times M
$$

In what follows, we highlight two properties of $\operatorname{VBTV}_{w}^{N L}$ that will be useful in the next Section where we consider variational models based on $\mathrm{VBTV}_{w}^{N L}$. 


\section{Properties of $\mathrm{VBTV}_{w}^{N L}$ :}

Let us first notice that $\operatorname{VBTV}_{w}^{N L}$ is a sup of linear forms

$$
J_{\eta}: \varphi \longmapsto \int_{M}\left(\varphi, \nabla_{w}^{N L^{*}} \eta\right)_{h} d M \quad \text { s.t. } \eta \in \mathcal{H}_{1}
$$

which are continuous with respect to the weak topology of $L^{2}(E)$ since they are bounded. Hence, for $\varphi \in L^{2}(E)$ and $\varphi_{n} \rightarrow \varphi$, we have $J_{\eta}\left(\varphi_{n}\right) \rightarrow J_{\eta}(\varphi)$.

\section{Lower semi-continuity}

We have

$$
J_{\eta}(\varphi)=\lim _{n} J_{\eta}\left(\varphi_{n}\right) \leq \liminf _{n} V B T V_{w}^{N L}\left(\varphi_{n}\right)
$$

and taking the sup over the set $\mathcal{H}_{1}$ yields

$$
V B T V_{w}^{N L}(\varphi) \leq \lim \inf _{n} \operatorname{VBTV} V_{w}^{N L}\left(\varphi_{n}\right)
$$

meaning that $V B T V_{w}^{N L}$ is lower semi-continuous with respect to the weak topology of $L^{2}(E)$.

2. Convexity The functional $V B T V_{w}^{N L}$ is convex as the supremum of the convex (since linear) functionals $J_{\eta}$. Indeed, $\forall \varphi_{1}, \varphi_{2} \in L^{1}(E)$, we have

$$
\begin{aligned}
J_{\eta}\left(t \varphi_{1}+(1-t) \varphi_{2}\right) & =t J_{\eta}\left(\varphi_{1}\right)+(1-t) J_{\eta}\left(\varphi_{2}\right) \\
& \leq t V B T V_{w}^{N L}\left(\varphi_{1}\right)+(1-t) V B T V_{w}^{N L}\left(\varphi_{2}\right)
\end{aligned}
$$

and consequently

$$
V B T V_{w}^{N L}\left(t \varphi_{1}+(1-t) \varphi_{2}\right) \leq t V B T V_{w}^{N L}\left(\varphi_{1}\right)+(1-t) V B T V_{w}^{N L}\left(\varphi_{2}\right)
$$

\section{Variational models for image processing tasks}

\subsection{Image features regularization}

3.1.1. Previous work In [20], Gilboa and Osher developed a nonlocal variational formulation based on the so-called anisotropic nonlocal total variation for the regularization of gray-level images $u_{0}: \Omega \subset \mathbb{R}^{2} \longrightarrow \mathbb{R}$, that can be written as follows with our notations

$$
\underset{u}{\arg \min } \frac{\lambda}{2}\left\|u-u_{0}\right\|_{L^{2}}^{2}+\left\|\nabla_{w}^{N L} u\right\|_{L^{1}}
$$


for $\lambda>0$, where $\left\|\nabla_{w}^{N L} u\right\|_{L^{1}}$ is of the form (2) for $n=1$.

In [5], we showed that we can derive an expression of the unique solution of the problem (25), and of its vectorial extension, using the dual formulation $V T V_{w}^{N L}$ of the term $\left\|\nabla_{w}^{N L}\right\|_{L^{1}}$, defined in (4).

More precisely, we considered the following variational problem

$$
\underset{u \in L^{2} \cap B V_{w}^{N L}\left(\Omega ; \mathbb{R}^{n}\right)}{\arg \min } \frac{\lambda}{2}\left\|u-u_{0}\right\|_{L^{2}}^{2}+V T V_{w}^{N L}(u)
$$

and showed that its unique solution $\underline{u}$ is of the form

$$
\underline{\mathrm{u}}=u_{0}-P_{K_{1 / \lambda}} u_{0}
$$

where $P$ is the projection operator, and $K_{1 / \lambda}$ is the closure in $L^{2}\left(\Omega ; \mathbb{R}^{n}\right)$ of the set $\mathcal{K}_{1 / \lambda}$ defined in (7).

3.1.2. The proposed model and its solutions Let us first point out that the variational problem (26) can be rewritten in the vector bundle context proposed in this paper when viewing a $\mathbb{R}^{n}$-valued function on $\Omega$ as a section of a (trivial) vector bundle over $\Omega$, and considering the regularizing term $V T V_{w}^{N L}$ in (26) as the (vector bundle) nonlocal total variation $V B T V_{w}^{N L}$ (18) induced by the trivial covariant derivative and the vector bundle metric consisting in the Euclidean scalar product in each fiber.

The following proposition shows that the problem (26) extends in a straightforward way when replacing the trivial covariant derivative by a non trivial one, assuming that this latter is compatible with a vector bundle positive definite metric.

Proposition 3.1. Let $E$ be a vector bundle over a complete Riemannian manifold $(M, g)$ equipped with a positive definite metric $h$ and a covariant derivative $\nabla$ compatible with $h$, and $u_{0} \in L^{2}(E)$. The unique solution $\underline{u}$ of the variational problem

$$
\underset{u \in L^{2} \cap B V_{w}^{N L}(E)}{\arg \min } \frac{\lambda}{2}\left\|u-u_{0}\right\|_{L^{2}}^{2}+V B T V_{w}^{N L}(u)
$$

$i s$

$$
\underline{u}=u_{0}-P_{K_{1 / \lambda}} u_{0}
$$

where $P$ is the projection operator, and $K_{1 / \lambda}$ is the closure in $L^{2}(E)$ of the set $\mathcal{K}_{1 / \lambda}$ defined in (21). 
Proof. As proved in Sect. 2, the functional $V B T V_{w}^{N L}$ is lower semicontinuous convex, and is also proper. Hence, the regularization model (28) is the proximity operator of $\frac{1}{\lambda} V B T V_{w}^{N L}$ evaluated at the element $u_{0}$. Then, according to Moreau's decomposition formula (see. e.g. [32] for details), we have

$$
\underline{u}=u_{0}-\frac{1}{\lambda} \operatorname{prox}\left(\lambda V B T V_{w}^{N L^{*}}\right)\left(\lambda u_{0}\right)
$$

By definition, $V B T V_{w}^{N L}$ is the support function of the closure $K_{1}$ of the convex set $\mathcal{K}_{1}$. Hence its convex conjugate $V B T V_{w}^{N L^{*}}$ is the indicator function $\delta_{K_{1}}$ of $K_{1}$. We then have

$$
\begin{aligned}
\operatorname{prox}\left(\lambda V B T V_{w}^{N L^{*}}\right)\left(\lambda u_{0}\right): & =\underset{v}{\arg \min } \frac{1}{2 \lambda}\left\|v-\lambda u_{0}\right\|_{L^{2}(E)}^{2}+\delta_{K_{1}}(v) \\
& =\underset{v \in K_{1}}{\arg \min }\left\|v / \lambda-u_{0}\right\|_{L^{2}(E)}^{2} \\
& =\lambda \underset{v \in K_{1 / \lambda}}{\arg \min }\left\|v-u_{0}\right\|_{L^{2}(E)}^{2} \\
& =\lambda P_{K_{1 / \lambda}} u_{0}
\end{aligned}
$$

Together with (30), it follows formula (29).

3.1.3. Algorithm to solving the proposed model The original problem (28) has the following primal-dual formulation

$$
\min _{u \in L^{2}(E)} \max _{\eta \in \mathcal{H}_{1}} \mathcal{L}(u, \eta):=\frac{\lambda}{2}\left\|u-u_{0}\right\|_{L^{2}}^{2}+\left(u, \nabla_{w}^{N L^{*}} \eta\right)
$$

The function $\mathcal{L}$ is strictly convex in $u$ and concave in $\eta$, which guarantees that the saddle points of $\mathcal{L}$ are of the form $(\underline{u}, \eta)$, where $\underline{u}$ is the unique solution of the primal problem (28) and $\eta$ is a solution of its dual formulation. In the discrete setting, the problem $(3 \overline{1})$ can be rewritten

$$
\min _{u \in L^{2}(E)} \max _{\eta \in \mathcal{H}_{1}} \frac{\lambda}{2}\left\|u-u_{0}\right\|_{L^{2}}^{2}+\left\langle\nabla_{w}^{N L} u, \eta\right\rangle,
$$

which is equivalent to

$$
\min _{u \in L^{2}(E)} \max _{\eta \in \Gamma\left(p r_{1}(E)\right)}\left\langle\nabla_{w}^{N L} u, \eta\right\rangle+\frac{\lambda}{2}\left\|u-u_{0}\right\|_{L^{2}}^{2}-\delta_{\mathcal{H}_{1}}(\eta)
$$

Problem (32) is of the form 


$$
\min _{u \in X} \max _{\eta \in Y}\langle A u, v\rangle+G(u)-F^{*}(v)
$$

where $X, Y$ are two finite-dimensional real vector spaces equipped with an inner product, the map $A: X \longrightarrow Y$ is a continuous linear operator possessing an adjoint $A^{*}$, the maps $G: X \longrightarrow[0,+\infty], F^{*}: Y \longrightarrow[0,+\infty]$ are proper, convex, lower semi-continuous, and $F^{*}$ is the convex conjugate of a convex lower semi-continuous function $F$. Then, the solutions of these problems satisfy

$$
\begin{array}{r}
A \underline{u} \in \partial F^{*}(\underline{\eta}) \\
-A^{*} \underline{\eta} \in \partial G^{*}(\underline{u})
\end{array}
$$

where $\partial$ denotes the sub-differential operator.

The problem (33) can be solved through one of the primal-dual algorithms presented in Chambolle and Pock [14]. Dealing with the ArrowHurwicz approach with fixed step sizes, the algorithm to solving (31) reads

* Initialization: Choose $\tau, \sigma>0$ s.t. $\tau \sigma\|A\|^{2}<4,\left(u^{0}, \eta^{0}\right) \in L^{2}(E) \times$ $\Gamma\left(p r_{1}(E)\right)$.

* Iterations $(n \geq 0)$ : Update $u^{n}, \eta^{n}$ as follows:

$$
\left\{\begin{array}{l}
\eta^{n+1}=\left(I+\sigma \partial F^{*}\right)^{-1}\left(\eta^{n}+\sigma A u^{n}\right) \\
u^{n+1}=(I+\tau \partial G)^{-1}\left(u^{n}-\tau A^{*} \eta^{n+1}\right)
\end{array}\right.
$$

In the context of problem (31), the iterative procedure is then the following

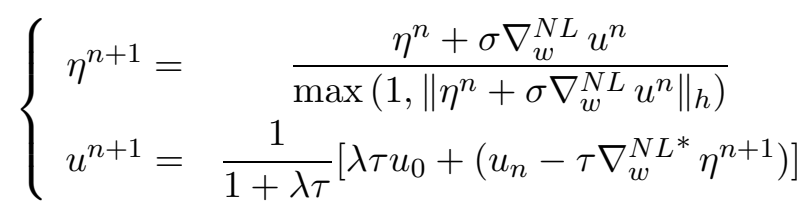

The convergence of the algorithm towards a saddle point is guaranteed by the condition $\tau \sigma\|A\|^{2}<4$ and is proved in [22].

\subsection{Image features enhancement}

3.2.1. Previous work In [7], Bertalmío et al. proposed a variational model for enhancing the local contrast of a color image $u_{0}=\left(u_{0}^{1}, u_{0}^{2}, u_{0}^{3}\right)$ based on some properties of the human visual system, that can be written as follows with our notations

$$
\underset{u^{k}}{\arg \min } \frac{\lambda}{2}\left\|u^{k}-u_{0}^{k}\right\|_{L^{2}}^{2}-\left\|\nabla_{w}^{N L} u^{k}\right\|_{L^{1}}, \quad k=1,2,3
$$


for $\lambda>0$, and where $\left\|\nabla_{w}^{N L} u\right\|_{L^{1}}$ is of the form (2) for $n=1$.

They showed that the problem (38) has a solution in the discrete case, i.e. when the image domain $\Omega$ is considered as discrete. Moreover, in order to approximate a solution, they considered a regularized version of the problem by regularizing the terms $\left\|\nabla_{w}^{N L} u^{k}\right\|_{L^{1}}$ and performed a gradient descent until reaching a steady-state.

In [5], we showed that we can derive an expression of the solutions of the original problem (38) and its vectorial extension by the use of the dual formulation $V T V_{w}^{N L}(u)$ of the term $\left\|\nabla_{w}^{N L} u\right\|_{L^{1}}$ defined in (4) with $n=3$, i.e. by considering the model

$$
\underset{u}{\arg \min } \frac{\lambda}{2}\left\|u-u_{0}\right\|_{L^{2}}^{2}-V T V_{w}^{N L}(u)
$$

assuming that the solutions belong to the discrete space $L^{2} \cap B V_{w}^{N L}\left(\Omega ; \mathbb{R}^{3}\right)$. We then showed that the solutions $\underline{u}$ are of the form

$$
\underline{u}=u_{0}-\underset{u^{*} \in \mathcal{K}_{1 / \lambda}}{\arg \max }\left\|u_{0}-u^{*}\right\|_{L^{2}}^{2}
$$

and $\mathcal{K}_{1 / \lambda}$ is defined in (7).

3.2.2. The proposed model and its solutions As the variational problem (26), the variational problem (39) can be rewritten in the vector bundle context proposed in this paper when viewing a $\mathbb{R}^{n}$-valued function on $\Omega$ as a section of a (trivial) vector bundle over $\Omega$, and considering the regularizing term $V T V_{w}^{N L}$ in (39) as the (vector bundle) nonlocal total variation $V B T V_{w}^{N L}$ (18) induced by the trivial covariant derivative and the vector bundle metric consisting in the Euclidean scalar product in each fiber.

The following proposition shows that the problem (39) extends in a straightforward way when replacing the trivial covariant derivative by a non trivial one, assuming that this latter is compatible with a vector bundle positive definite metric.

Proposition 3.2. Let $E$ be a vector bundle over a discrete Riemannian manifold $(M, g)$, equipped with a positive definite metric $h$ and a covariant derivative $\nabla$ compatible with $h$, and $u_{0} \in L^{2}(E)$. The solutions $\underline{u}$ of the variational problem

$$
\underset{u \in L^{2} \cap B V_{w}^{N L}(E)}{\arg \min } \frac{\lambda}{2}\left\|u-u_{0}\right\|_{L^{2}}^{2}-V B T V^{N L}(u)
$$


are

$$
\underline{u}=u_{0}-\underset{u^{*} \in \mathcal{K}_{1 / \lambda}}{\arg \max }\left\|u_{0}-u^{*}\right\|_{L^{2}}^{2}
$$

where $\mathcal{K}_{1 / \lambda}$ is defined in (21).

Proof. The problem (41) is of the form

$$
\inf _{u \in X}\{G(u)-F(u)\}
$$

where $F$ and $G$ are two lower semi-continuous functionals on a reflexive Banach space $X$ such that $F$ is convex and $G$ satisfies $G(u) /\|u\| \rightarrow \infty$ as $\|u\| \rightarrow \infty$. Then according to Theorem 2.7 in [36], if $\underline{u}^{*}$ is a solution of the dual problem

$$
\inf _{u^{*} \in X^{*}}\left\{F^{*}\left(u^{*}\right)-G^{*}\left(u^{*}\right)\right\}
$$

where $F^{*}$ resp. $G^{*}$ denotes the convex conjugate of $F$ resp. $G$, then there exists a solution $\underline{\mathrm{u}}$ of the primal problem (43), and the solutions of both problems are connected by the formulas

$$
\begin{aligned}
& F(\underline{\mathrm{u}})+F^{*}\left(\underline{\mathrm{u}}^{*}\right)=\left\langle\underline{\mathrm{u}}, \underline{\mathrm{u}}^{*}\right\rangle \\
& G(\underline{\mathrm{u}})+G^{*}\left(\underline{\mathrm{u}}^{*}\right)=\left\langle\underline{\mathrm{u}}, \underline{\mathrm{u}}^{*}\right\rangle .
\end{aligned}
$$

Hence, the problem (41) can be solved through its dual problem (44).

The convex conjugate of the functional

$$
G: u \longmapsto \frac{\lambda}{2}\left\|u-u_{0}\right\|_{L^{2}}^{2}
$$

is

$$
G^{*}: u^{*} \longrightarrow \frac{1}{2 \lambda}\left(\left\|u^{*}\right\|_{L^{2}}^{2}+2\left\langle u^{*}, \lambda u_{0}\right\rangle_{L^{2}}\right)
$$

As the manifold $M$ is discrete, the set $\mathcal{K}_{1}$ is close and convex, hence the functional $F:=V B T V_{w}^{N L}$ is the support function of $\mathcal{K}_{1}$, and its convex conjugate is the indicator function $\chi_{\mathcal{K}_{1}}$ of $\mathcal{K}_{1}$. Then, the dual problem (44) reads

$$
\underset{u^{*} \in L^{2}(E)}{\arg \min } \quad \chi \mathcal{K}_{1}\left(u^{*}\right)-\frac{1}{2 \lambda}\left[\left\|u^{*}\right\|_{L^{2}}^{2}+2\left\langle u^{*}, \lambda u_{0}\right\rangle_{L^{2}}\right]
$$


Since the $L^{2}$ norm of sections in $\mathcal{K}_{1}$ is bounded, it is clear that the functional to minimize in (48) is coercive as well as bounded from below. Then, since $L^{2}(E)$ is reflexive, we deduce that from any minimizing sequence $\underline{u}_{n}{ }^{*}$ for the functional in (48) can be extracted a subsequence ${\underline{u_{n_{j}}}}^{*}$ converging weakly towards some $\underline{u}^{*} \in L^{2}(E)$. Finally, as we are in a discrete space, this sequence is actually strongly converging towards $\underline{u}^{*}$, showing that $\underline{u}^{*}$ is a solution of problem (48).

The dual problem can then be rewritten

$$
\underline{u}^{*}=\underset{u^{*} \in \mathcal{K}_{1}}{\arg \max }\left\|u^{*}\right\|_{L^{2}}^{2}+2\left\langle u^{*}, \lambda u_{0}\right\rangle_{L^{2}}
$$

Adding the term $\left\|\lambda u_{0}\right\|_{L^{2}}$ does not affect the solution of the problem (49), hence we can write

$$
\underline{u}^{*}=\underset{u^{*} \in \mathcal{K}_{1}}{\arg \max }\left\|u^{*}+\lambda u_{0}\right\|_{L^{2}}^{2}
$$

which is equivalent to

$$
\underline{u}^{*}=-\underset{u^{*} \in \mathcal{K}_{1}}{\arg \max }\left\|\lambda u_{0}-u^{*}\right\|_{L^{2}}^{2}
$$

Finally, we deduce from (46) and (47) that the solutions $\underline{u}$ of the original problem (41) are

$$
\underline{u}=u_{0}-\frac{1}{\lambda} \underset{u^{*} \in \mathcal{K}_{1}}{\arg \max } \quad\left\|\lambda u_{0}-u^{*}\right\|_{L^{2}(E)}^{2}
$$

which can be rewritten

$$
\underline{u}=u_{0}-\underset{u^{*} \in \mathcal{K}_{1 / \lambda}}{\arg \max }\left\|u_{0}-u^{*}\right\|_{L^{2}(E)}^{2}
$$

3.2.3. Algorithm to solving the proposed model As we are in a discrete space, the problem (41) can be rewritten as

$$
\underset{u \in L^{2} \cap B V_{w}^{N L}(E)}{\arg \min } \frac{\lambda}{2}\left\|u-u_{0}\right\|_{L^{2}(E)}^{2}-\left\|\nabla_{w}^{N L} u\right\|_{L^{1}}
$$

making it be of the form

$$
\min _{u \in X} G(u)-F(A u)
$$


with $A: X \longrightarrow Y$ is a linear operator between two finite dimensional vector spaces, and possessing an adjoint operator $A^{*}$.

Then, as demonstrated in [36] and already mentioned in Sect. 3.2.2, the problem (51) has the following dual formulation

$$
\min _{u \in X^{*}}(F \circ A)^{*}(u)-G^{*}(u)
$$

which is equivalent to

$$
\min _{\eta \in Y^{*}} F^{*}(\eta)-G^{*}\left(A^{*} \eta\right)
$$

by definition of $F$.

Hence, the solutions $\underline{u}$ and $\eta$ of the primal (51) and dual (53) problems are linked by the following formulae

$$
\begin{aligned}
& A \underline{\mathrm{u}} \in \partial F^{*}\left(\underline{\eta}^{*}\right) \\
& A^{*} \underline{\eta}^{*} \in \partial G(\underline{\mathrm{u}})
\end{aligned}
$$

Our proposal is then to adapt the Arrow-Hurwicz algorithm (37) to the equations (54) and (55). It gives the following iterative scheme

$$
\left\{\begin{array}{l}
\eta^{n+1}=\frac{\eta^{n}+\sigma \nabla_{w}^{N L} u^{n}}{\max \left(1,\left\|\eta^{n}+\sigma \nabla_{w}^{N L} u^{n}\right\|_{h}\right)} \\
u^{n+1}=\frac{1}{1+\lambda \tau}\left[\lambda \tau u_{0}+\left(u_{n}+\tau \nabla_{w}^{N L^{*}} \eta^{n+1}\right)\right]
\end{array}\right.
$$

\section{Applications to local contrast modification}

\subsection{The chosen nonlocal covariant derivative and its numerical implementation}

4.1.1. The chosen local covariant derivative In [3], Batard and Sochen constructed a covariant derivative $\nabla^{o p t}$ compatible with a vector bundle metric as solution of a minimization problem, and apply the heat diffusion of the corresponding Laplacian for color image regularization purpose.

4.1.1.1. The construction of the covariant derivative The idea developed in [3] is to consider a color image $u=\left(u^{1}, u^{2}, u^{3}\right)$ defined on a domain $\Omega \subset \mathbb{R}^{2}$ as a section of a (trivial) vector bundle $E$ of rank 3 over $\Omega$. Taking the Euclidean scalar product in each fiber as a positive definite metric $h$ on $E$, a covariant derivative compatible with $h$ is completely determined by a 
connection 1-form $\omega \in \Gamma\left(T^{*} \Omega \otimes \mathfrak{s o}(3)\right)$. Then, considering $E$ as an associated bundle $P \times{ }_{(\rho, \mathrm{SO}(3))} \mathbb{R}^{3}$ where $P$ is the principal bundle $\Omega \times \mathrm{SO}(3)$ over $\Omega$ and $\rho$ is the standard representation of $\mathrm{SO}(3)$ on $\mathrm{GL}\left(\mathbb{R}^{3}\right)$, a connection 1-form on $E$ is determined by a horizontal bundle $H P$ on $P$, which is a subbundle of dimension 2 of $P$.

The authors proposed a variational model in order to construct an optimal horizontal bundle $H P^{o p t}$ of $P$. The optimal horizontal bundle is the horizontal bundle such that the Riemannian metric $h^{\text {opt }}$ on $P$ that makes it be orthogonal to the vertical bundle of $P$ minimizes the elastic potential energy of the graph of $u$ (formally, the Riemannian metric $h^{\text {opt }}$ minimizes the Dirichlet energy of the graph of $u$ in $\left.\Omega \times \mathrm{SO}(3) \times \mathbb{R}^{3}\right)$. Intuitively, the metric $h^{\text {opt }}$ can be viewed as the one that makes the Riemannian geometry of the graph of $u$ be the most consistent with respect to the Riemannian geometry of the ambient space $\Omega \times \mathrm{SO}(3) \times \mathbb{R}^{3}$ (see [3] for details).

Finally, they showed that the corresponding optimal connection 1-form $\omega^{o p t}$ is given by the matrix field coefficients

$$
\begin{aligned}
\omega_{12}^{o p t}= & -\sum_{k=1}^{2} \alpha \kappa\left((b e-d c)\left(u_{x_{l}}^{2} u^{3}-u_{x_{l}}^{3} u^{2}\right)+(b c-a e)\left(u_{x_{l}}^{1} u^{3}-u_{x_{l}}^{3} u^{1}\right)\right. \\
& \left.+\left(a d-b^{2}\right)\left(u_{x_{l}}^{1} u^{2}-u_{x_{l}}^{2} u^{1}\right)\right) d x_{l} \\
\omega_{13}^{o p t}= & -\sum_{k=1}^{2} \alpha \kappa\left((c e-f b)\left(u_{x_{l}}^{2} u^{3}-u_{x_{l}}^{3} u^{2}\right)+\left(a f-c^{2}\right)\left(u_{x_{l}}^{1} u^{3}-u_{x_{l}}^{3} u^{1}\right)\right. \\
& \left.+(b c-a e)\left(u_{x_{l}}^{1} u^{2}-u_{x_{l}}^{2} u^{1}\right)\right) d x_{l} \\
\omega_{23}^{o p t}= & -\sum_{k=1}^{2} \alpha \kappa\left(\left(f d-e^{2}\right)\left(u_{x_{l}}^{2} u^{3}-u_{x_{l}}^{3} u^{2}\right)+(c e-f b)\left(u_{x_{l}}^{1} u^{3}-u_{x_{l}}^{3} u^{1}\right)\right. \\
& \left.+(b e-d c)\left(u_{x_{l}}^{1} u^{2}-u_{x_{l}}^{2} u^{1}\right)\right) d x_{l}
\end{aligned}
$$

where

$$
\begin{gathered}
a=\delta+\kappa\left[\left(u^{2}\right)^{2}+\left(u^{3}\right)^{2}\right] \quad b=\kappa u^{1} u^{2} \quad c=-\kappa u^{1} u^{3} \\
d=\delta+\kappa\left[\left(u^{1}\right)^{2}+\left(u^{3}\right)^{2}\right] \quad e=\kappa u^{2} u^{3} \quad f=\delta+\kappa\left[\left(u^{1}\right)^{2}+\left(u^{2}\right)^{2}\right]
\end{gathered}
$$

and

$$
\alpha=\frac{1}{2 c b e+f a d-a e^{2}-f b^{2}-c^{2} d}
$$

for some $\kappa, \delta>0$. 


\subsubsection{Heat diffusion of generalized Laplacian for image regularization}

Given a vector bundle $E$ over a Riemannian manifold $(M, g)$ equipped with a positive definite metric $h$ and a covariant derivative $\nabla$ compatible with $h$, we showed in [4] that the gradient descent of the variational problem

$$
\underset{u}{\arg \min } \int_{M}\|\nabla u\|_{g^{-1} \otimes h}^{2} d M
$$

is a generalized heat equation

$$
\frac{\partial u}{\partial t}+\nabla^{*} \nabla u=0
$$

where $\nabla^{*}$ is the adjoint of $\nabla$.

In [3], the heat diffusion (58) associated to the covariant derivative $\nabla^{o p t}=d+\omega^{o p t}$ is applied to a color image $u=\left(u^{1}, u^{2}, u^{3}\right)$ given in the RGB color space, where the domain $\Omega$ is equipped with the following Riemannian metric

$$
g:=\left(\begin{array}{cc}
1+\kappa \sum_{k=1}^{3}\left(u_{x_{1}}^{k}\right)^{2} & \kappa \sum_{k=1}^{3} u_{x_{1}}^{k} u_{x_{2}}^{k} \\
\kappa \sum_{k=1}^{3} u_{x_{1}}^{k} u_{x_{2}}^{k} & 1+\kappa \sum_{k=1}^{3}\left(u_{x_{2}}^{k}\right)^{2}
\end{array}\right)
$$

in the frame $\left(\partial / \partial x_{1}, \partial / \partial x_{2}\right)$, and $\kappa$ is the constant that appears in the expression of $\omega^{\text {opt }}$ above. The aim of the metric (59) is to make the diffusion take into account the image edges.

Fig. 1 reproduces some of the results available in [3]. It compares the heat diffusion of the operator $\nabla^{o p t *} \nabla^{o p t}$ to the heat diffusion of the operator $\nabla^{t r i}{ }^{*} \nabla^{t r i}$ associated to the trivial covariant derivative $\nabla^{t r i}$ and the Riemannian metric (59), that corresponds to the Laplace-Beltrami operator diffusion introduced in [35]. The same parameters have been taken in both cases, i.e. $\kappa=0.0005, d t=0.1, \delta=1$, and the diffusions were stopped after 50 iterations. We observe that the heat diffusion based on the optimal covariant derivative preserves more the small details of the original image like the textures and tends also to preserve more its colors.

4.1.1.3. Interpretation of the results Under the identification between a section $u$ of the associated bundle $P \times_{(\rho, \mathrm{SO}(3))} \mathbb{R}^{3}$ and a $\mathrm{SO}(3)$-equivariant function $f_{u}$ on the principal bundle $P$, the heat diffusion

$$
\frac{\partial u}{\partial t}+\nabla^{* o p t} \nabla^{o p t} u=0
$$


that we consider in order to regularize an image $u_{\mid t=0}$ writes

$$
\frac{\partial f_{u}}{\partial t}+\left(\Delta_{h^{o p t}} \otimes 1+1 \otimes C a s\right) f_{u}=0
$$

where Cas is a Casimir operator, which corresponds to the linear operator $-2 I d$ in our case, and $\Delta_{h^{o p t}}$ is the Laplace-Beltrami operator associated to the Riemannian metric $h^{\text {opt }}$.

The identification between the heat diffusion (60) and the heat diffusion (61) gives us more insight about the behavior of (60). Indeed, as a diffusion with respect to a Laplace-Beltrami operator (up to the 0 order differential operator Cas), we know that (61) tends to smooth the initial function $f_{u \mid t=0}$ in an anisotropic way with respect to $h^{\text {opt }}$. More precisely, it tends to smooth the image in the directions of the eigenvector fields associated to the smallest eigenvalues of $h^{\text {opt }}$ and block the diffusion in the directions of the eigenvector fields associated to its highest eigenvalues.

Unlike the standard image regularization models, the diffusion (61) does not only take into account the color differences between neighboring pixels in the image domain, but also the color differences due to the derivative of $f_{u}$ with respect to its $\mathrm{SO}(3)$ coordinates. Hence, if the color differences at one pixel are large in every directions, which is determined by the metric $h^{o p t}$, then the diffusion will be blocked in that pixel. In particular, the norm of the derivative of $f_{u}$ at some pixel with respect to its $\mathrm{SO}(3)$ coordinates has more chance to be large if the pixel value is far from the origin $(0,0,0)$ which corresponds to colors with high luminance and saturation when dealing with the $R G B$ color space. As a consequence, the diffusion (60) may perserve structures of high luminance and saturation, which would explain the behavior observed in Fig. 1.

4.1.2. Nonlocal extension of the chosen covariant derivative Based on the results obtained in [3] and showed in Fig. 1, we aim at using the optimal covariant derivative $\nabla^{o p t}$ in the context of the variational models described in this paper. To that purpose, we need to extend $\nabla^{o p t}$ in a nonlocal way by the use of the corresponding parallel transport map, as described in Sect. 2, and determine the symmetric function $w$.

4.1.2.1. The function $w$ The function $w$ completely determines the behavior of the models (28) and (41), meaning that the choice of $w$ is dependent of the application targeted. In this paper, we made the choice of applying the models (28) and (41) to local contrast reduction/enhancement and we then 


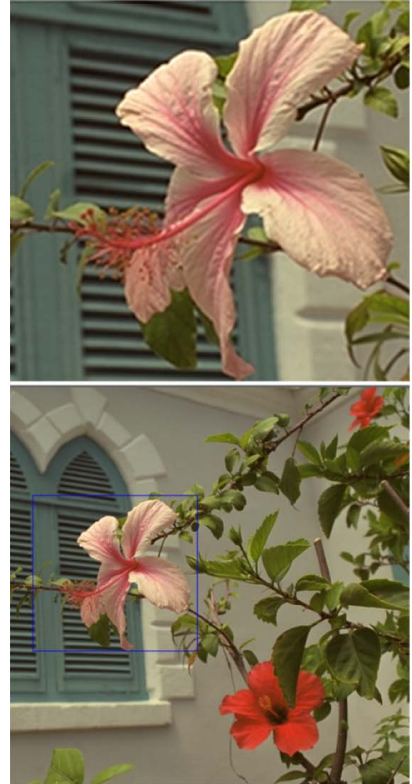

(a) Original image
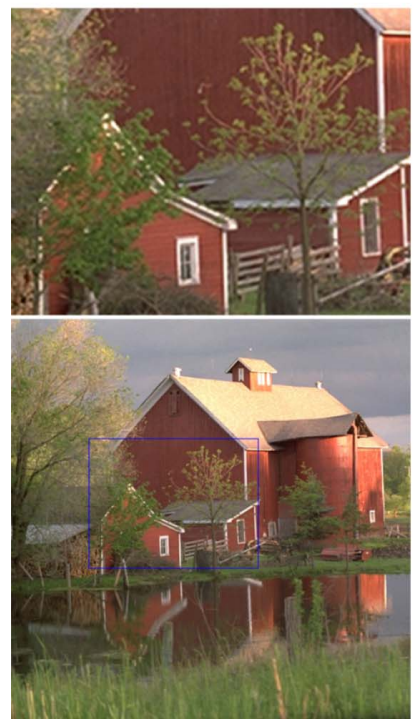

(d) Original image

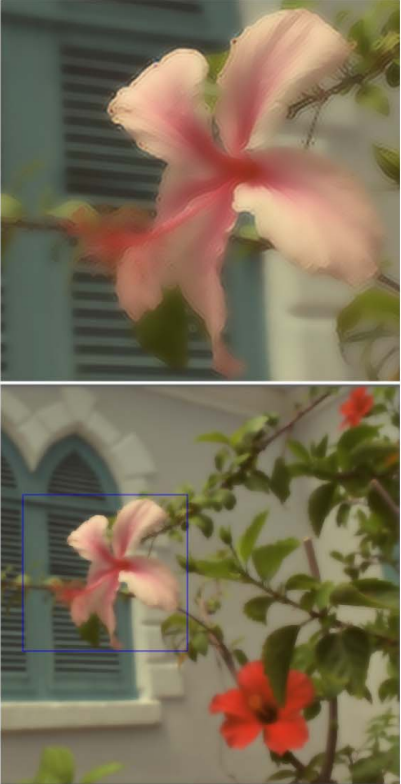

(b) Laplace-Beltrami
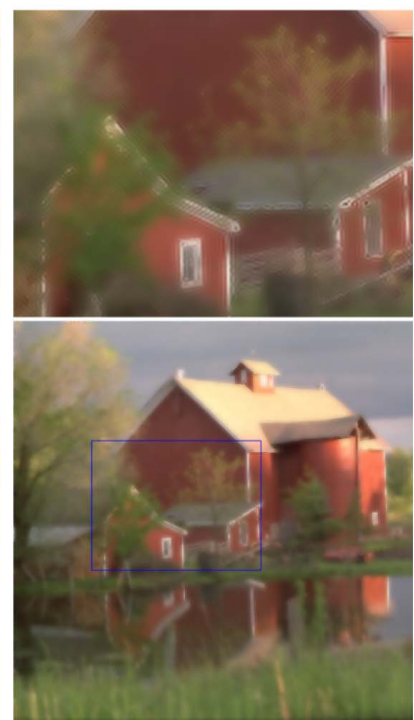

(e) Laplace-Beltrami

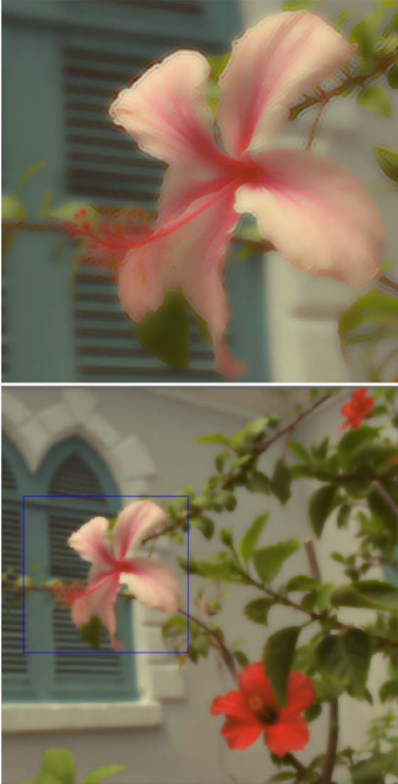

(c) Operator $\nabla^{o p t^{*}} \nabla^{o p t}$
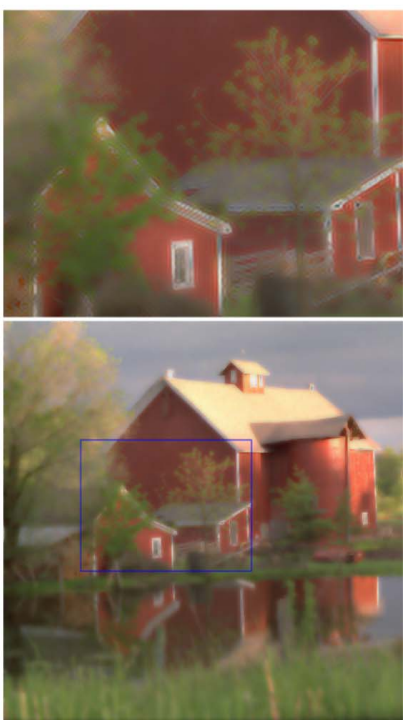

(f) Operator $\nabla^{o p t^{*}} \nabla^{o p t}$

Figure 1: Heat diffusions of generalized Laplacians (58). Comparison between the operator $\nabla^{o p t^{*}} \nabla^{o p t}$ and the Laplace-Beltrami operator associated to the Riemannian metric (59). Images taken from [3]. (Color figure online) 
choose $w$ as a truncated (normalized) Gaussian kernel with fixed variance value, but varying the window size. The variance value we select is 2000 , which makes the kernel $w$ be almost flat.

4.1.2.2. The Riemannian metric on the domain $\Omega$ The Riemannian metric we equip the domain $\Omega$ of determines the geodesic curves along which we perform the parallel transport to determine the non-local covariant derivative (15) and its adjoint (17).

Unlike the local case in Sect. 4.1.1, we equip the image domain $\Omega$ with the Euclidean metric in the following experiments where we test the nonlocal models (28) and (41), which makes the geodesic curves in $\Omega$ be the straight lines. Indeed, in Sect. 4.1.1, as we were using the $L^{2}$ norm of the covariant derivative, we made use of the Riemannian metric (59) in order to guarantee the anisotropy of the diffusion with respect to the image edges. In the models (28) and (41), the anisotropy of the diffusion with respect to the edges is taken into account by the $L^{1}$ norm of the (nonlocal) covariant derivative.

4.1.2.3. Numerical accuracy of the parallel transport map Numerical computations show that the covariant derivative $\nabla^{\text {opt }}$ is not flat. Hence, there exists no local frame of $E$ in which its corresponding connection 1-form vanishes, i.e. that there exists no local frame of $E$ in which the parallel transport map associated to $\nabla^{\text {opt }}$ writes as the Identity map. As a consequence, we have to determine the corresponding parallel transport map by solving the following type of differential equation

$$
\nabla_{\dot{\gamma}(t)}^{o p t} u(t)=0, \quad u(0)=u(\gamma(0))
$$

i.e.

$$
d u(t)=-\omega^{o p t}(\dot{\gamma}(t))(u(t)), \quad u(0)=u(\gamma(0))
$$

Our proposal is then to make use of an explicit Euler scheme

$$
u\left(t_{n+1}\right)=u\left(t_{n}\right)-d t \omega^{o p t}\left(\dot{\gamma}\left(t_{n}\right)\right)\left(u\left(t_{n}\right)\right), \quad u\left(t_{0}\right)=u(\gamma(0))
$$

to solve (63) numerically.

The numerical accuracy of the scheme (64) depends on:

(i) the step $d t$, which is related to the discretization of the straight lines $\gamma$.

(ii) the way we approximate $\omega$ at the points $\gamma\left(t_{n}\right)$ of the discrete lines, as they do not necessarily correspond to pixel locations, which are the points where $\omega$ is constructed. 
Let $\left(x_{1}, x_{2}\right)$ and $\left(y_{1}, y_{2}\right)$ be two points in $\Omega$ corresponding to pixel locations, i.e. $x_{1}, x_{2}, y_{1}, y_{2} \in \mathbb{N}$. We compute the parallel transport of $u\left(y_{1}, y_{2}\right)$ along the straight line $\gamma$ joining $\left(y_{1}, y_{2}\right)$ and $\left(x_{1}, x_{2}\right)$ in the following manner:

(i) We discretize $\gamma$ with $\max \left(\left|y_{1}-x_{1}\right|,\left|y_{2}-x_{2}\right|\right)$ points. It gives

$$
d t=\frac{1}{\max \left(\left|y_{1}-x_{1}\right|,\left|y_{2}-x_{2}\right|\right)} \sqrt{\left(y_{1}-x_{1}\right)^{2}+\left(y_{2}-x_{2}\right)^{2}}
$$

and

$\gamma\left(t_{n}\right)=\left(y_{1}-n \frac{\left(y_{1}-x_{1}\right)}{\max \left(\left|y_{1}-x_{1}\right|,\left|y_{2}-x_{2}\right|\right)}, y_{2}-n \frac{\left(y_{2}-x_{2}\right)}{\max \left(\left|y_{1}-x_{1}\right|,\left|y_{2}-x_{2}\right|\right)}\right)$

(ii) We approximate the coefficients $w_{i j}^{o p t}\left(\dot{\gamma}\left(t_{n}\right)\right)$ of the matrix $w^{\text {opt }}\left(\dot{\gamma}\left(t_{n}\right)\right)$ at the point $\gamma\left(t_{n}\right)$, for $i, j=1, \cdots, 3$ and $n=0, \cdots, \max \left(\left|y_{1}-x_{1}\right|,\left|y_{2}-x_{2}\right|\right)$ in (64) as

$$
\begin{aligned}
& \frac{\left(y_{1}-x_{1}\right)}{\max \left(\left|y_{1}-x_{1}\right|,\left|y_{2}-x_{2}\right|\right)} \Upsilon_{1 i}^{j}\left(R\left(\gamma\left(t_{n}\right)\right)\right) \\
& +\frac{\left(y_{2}-x_{2}\right)}{\max \left(\left|y_{1}-x_{1}\right|,\left|y_{2}-x_{2}\right|\right)} \Upsilon_{2 i}^{j}\left(R\left(\gamma\left(t_{n}\right)\right)\right)
\end{aligned}
$$

where $R\left(\gamma\left(t_{n}\right)\right)$ denotes the rounding of each coordinate of $\gamma\left(t_{n}\right)$ to its nearest integer. In other words, we approximate the symbols $\Upsilon_{k l}^{j}, k=1,2$ at the points $\gamma\left(t_{n}\right)$ by $\Upsilon_{k l}^{j}\left(\widetilde{\gamma\left(t_{n}\right)}\right)$ where $\widetilde{\gamma\left(t_{n}\right)}$ is the closest point to $\gamma\left(t_{n}\right)$ that corresponds to a pixel location.

\subsection{Numerical implementation of the algorithms, its limits and solutions to overcome them}

In the following Sections, we test the algorithms (37) and (56) for local contrast reduction and local contrast enhancement respectively, with $\lambda=$ 0.1 . We test each algorithm with the trivial covariant derivative $\nabla^{t r i}$ as well as the optimal one $\nabla^{o p t}$ aforementioned for different window sizes, and where a color image is expressed in the $R G B$ color space equipped with the Euclidean metric. We choose constant values for the parameters $\tau$ and $\sigma$ ( $\tau=\sigma=0.1$ ) such that they satisfy the conditions $\tau \sigma\left\|\nabla_{w}^{N L}\right\|^{2}<4$ for both covariant derivatives in order to guarantee the convergence of the algorithms. Finally, the stopping criteria is

$$
\frac{1}{3|\Omega|}\left\|u_{n+1}-u_{n}\right\|_{L^{2}}<0.001
$$


The computational efficiency is the main drawback of the proposed algorithms, and they are not practical in their current state. For instance, it takes a couple of days for the algorithms to converge when the covariant derivative is the optimal one and the window size is $40 \times 40$. However, there are several options to speed them up:

1. We can use some acceleration techniques that are proposed in [14], e.g. updating the values of the parameters $\tau$ and $\sigma$ at each iteration. It will reduce the number of iterations until convergence, but still the algorithms would be slow for large window sizes as it takes time to execute one iteration due to the non-local aspect of the gradient operator and its adjoint. The situation is even worse when the covariant derivative is not flat, which is the case of our optimal covariant derivative, as it requires the use of a parallel transport map computed through an Euler scheme (64) to determine the non-local gradient and its adjoint operator at each point.

2. A more efficient strategy, but less straightforward would be to make use of the acceleration technique used by Bertalmío et al. [7] in the scalar case. Indeed, they approximate the numerical solution of the minimization problem (38) by the steady-state of the gradient descent associated to a differentiable approximation of the minimization problem. Then they reach the steady-state in seconds by computing the gradient at each iteration in a very fast manner thanks to the use of the fast Fourier transform. Our current work is devoted to adapt the strategy of Bertalmío et al. to our model.

\subsection{Local contrast reduction}

In Fig. 2-3, we show the solutions of the algorithm (37). For both covariant derivatives, we observe on Fig. $2-3$ that the window size influences the behavior of the regularizations, i.e. a small window size tends to make the regularization remove the details that were too small in the original images on Fig. 2-3(a) while reducing very few its contrast (Fig. 2-3(b-c)), whereas a larger window size produces a larger reduction of the contrast of the original images that smoothes the small details but preserves their aspect (e.g. textures) (see Fig. 2-3(f-g)), the amount of contrast reduction being correlated with the window size (compare Fig. 2-3(d-e) and Fig. 2-3(f-g)). An analysis of the behavior of the model is provided in Sect. 4.5.

Given a window size, the results on Fig. 2-3 confirm what we have observed in [3] with generalized heat diffusions (see Fig. 1), i.e. both covariant 


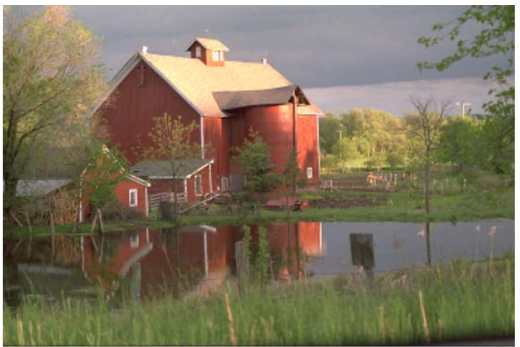

(a) Original image $u_{0}$
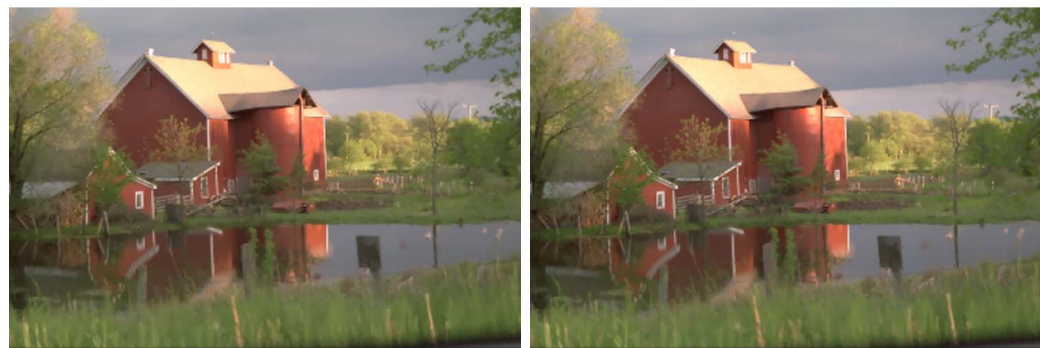

(b) NLCV induced by the trivial one with (c) NLCV induced by the optimal one window size $3 \times 3$ with window size $3 \times 3$
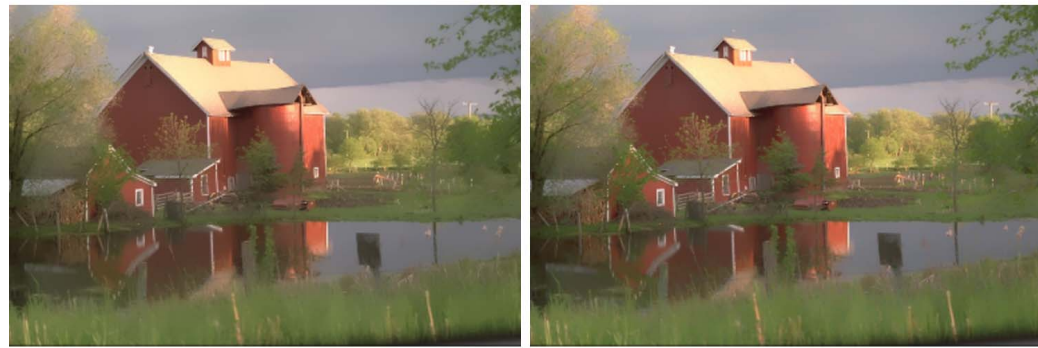

(d) NLCV induced by the trivial one with (e) NLCV induced by the optimal one window size $10 \times 10$ with window size $10 \times 10$
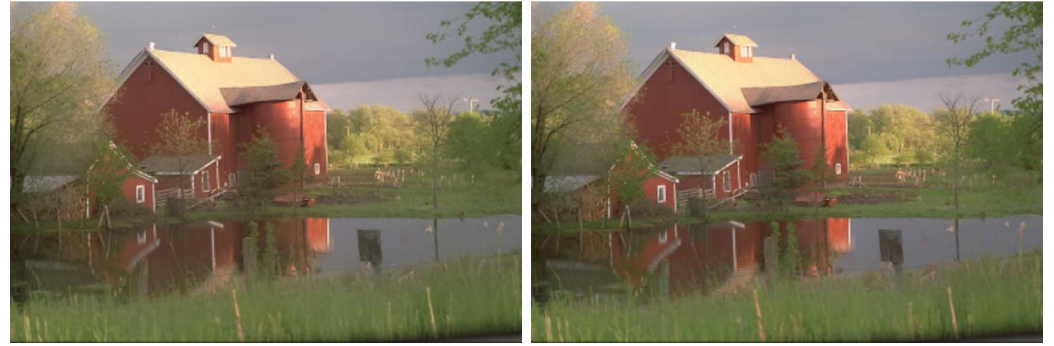

(f) NLCV induced by the trivial one with (g) NLCV induced by the optimal one window size $40 \times 40$ with window size $40 \times 40$

Figure 2: Solutions $\underline{u}$ (29) of the local contrast regularization model (28) for different nonlocal covariant derivatives (NLCV). (Color figure online) 


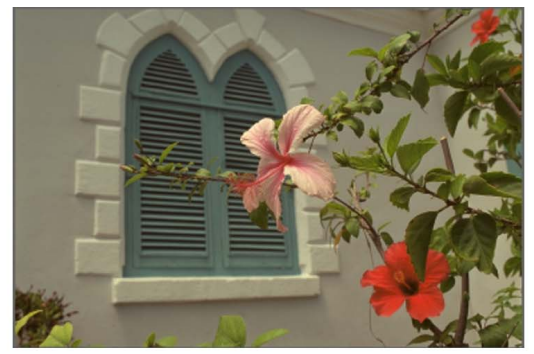

(a) Original image $u_{0}$
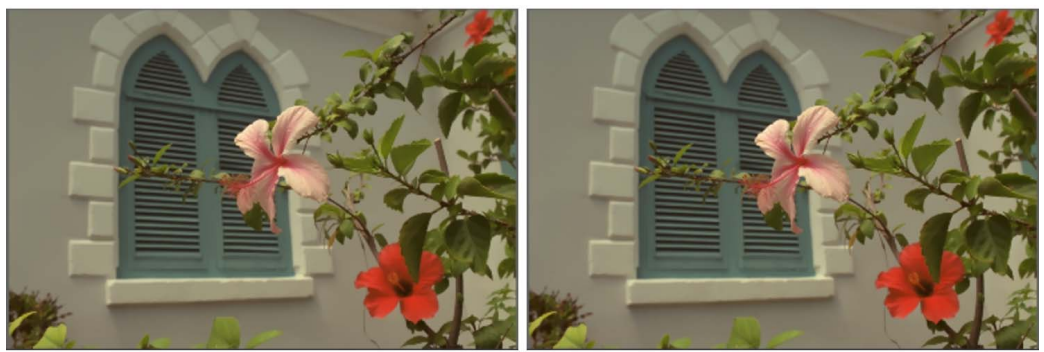

(b) NLCV induced by the trivial one with (c) NLCV induced by the optimal one window size $3 \times 3$ with window size $3 \times 3$
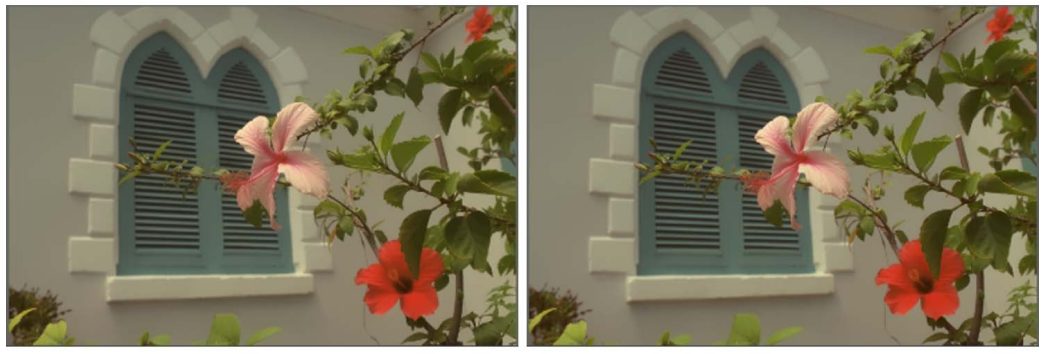

(d) NLCV induced by the trivial one with (e) NLCV induced by the optimal one window size $10 \times 10$ with window size $10 \times 10$
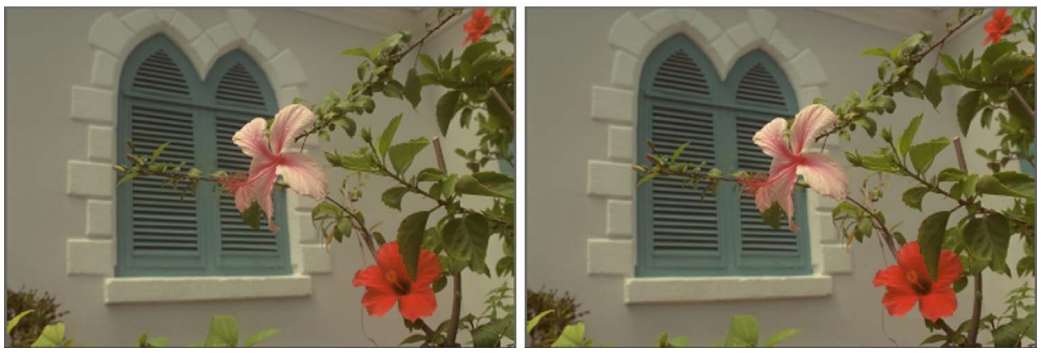

(f) NLCV induced by the trivial one with (g) NLCV induced by the optimal one window size $40 \times 40$ with window size $40 \times 40$

Figure 3: Solutions $\underline{u}$ (29) of the local contrast reduction model (28) for different nonlocal covariant derivatives (NLCV). (Color figure online) 
derivatives make the model reduce the noise and smooth small details of the original image like its textures, but the optimal covariant derivative makes the model smooth less these small details and produce more saturated colors, meaning that the model preserves more the aspect of the original image than when it is induced by the trivial covariant derivative (compare Fig. 2-3(f) and Fig. 2-3(g)).

In order to measure and compare the amount of contrast reduction of the images on Fig. 2-3(f) and Fig. 2-3(g), we propose to make use of the following mean contrast (MC) measure of their luminances

$$
\frac{1}{|\Omega|} \sum_{x} \sum_{y} w(x, y)\left|u_{l}(x)-u_{l}(y)\right|
$$

for $w$ being the truncated (normalized) Gaussian kernel of size $40 \times 40$ and variance 2000, and where we define the luminance $u_{l}$ of a color image $u=$ $\left(u^{1}, u^{2}, u^{3}\right)$ as $u_{l}=\frac{1}{3} \sum_{k=1}^{3} u^{k}$.

Fig. 4 shows the luminances of the original color images Fig. 2-3(a) and their contrast reduced versions on Fig. 2-3(f) and Fig. 2-3(g). We compute the mean contrast (66) of each luminance image, and the results show that the reduction of contrast is almost the same for both covariant derivatives.

Through the experiments conducted in this Section, we have shown that using the optimal covariant derivative makes our local contrast reduction model preserve more the aspect of the original image than the trivial covariant derivative does while reducing (almost) equally its contrast.

Finally, we show on Fig. 5 the plots of the energy (28) at different iterations of the algorithm (37) until convergence applied to the images on Fig. 2(a) and Fig. 3(a) and tested with different window sizes and the two covariant derivatives aforementioned.

\subsection{Local contrast enhancement}

In Fig. 6-7-8-9, we show solutions of the algorithm (56). Applying the enhancement model for window sizes that are too small amplifies the noise of the original images for both the trivial and the optimal covariant derivatives (see Fig. 6-8(b-c) for a window size $5 \times 5$ and Fig. 6-8(d-e) for a window size $10 \times 10$ ), and the noise amplification tends to reduce with the increase of the window size as we can observe on Fig. $6-8(\mathrm{f}-\mathrm{g})$ where a window size $40 \times 40$ has been taken. An analysis of the behavior of the model is provided in Sect. 4.5. Note that this is consistent with the results obtained in the previous Section where we showed that the regularization model smoothes 


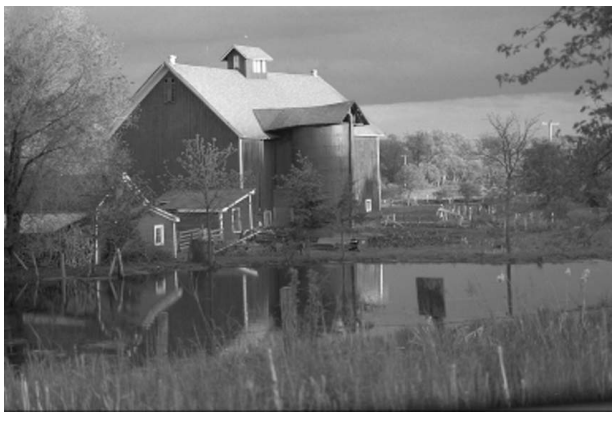

(a) Original image: MC 20.65

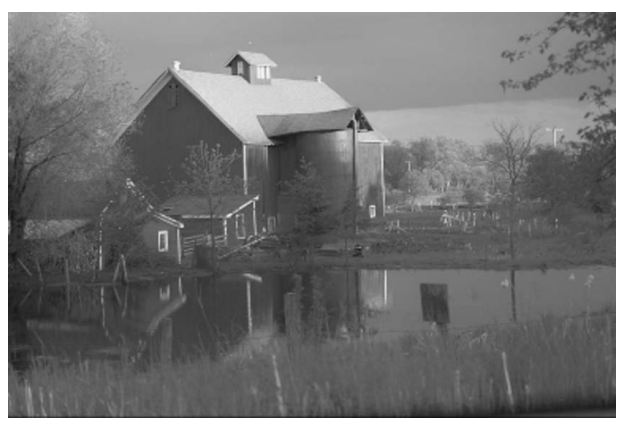

(c) NLCV induced by the trivial one with window size $40 \times 40$ : MC 16.22

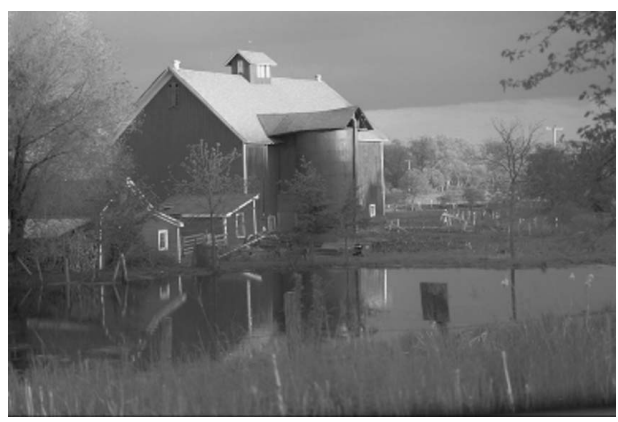

(e) NLCV induced by the optimal one with window size $40 \times 40$ : MC 16.34

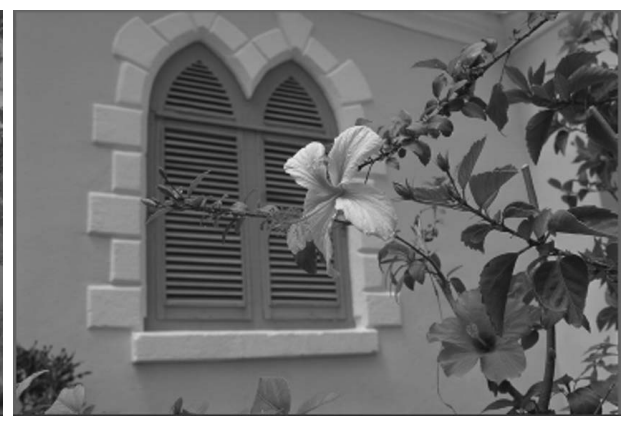

(b) Original image: MC 27.12

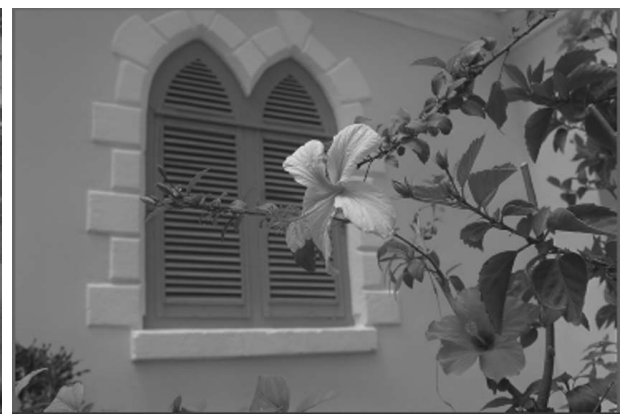

(d) NLCV induced by the trivial one with window size $40 \times 40$ : MC 20.68

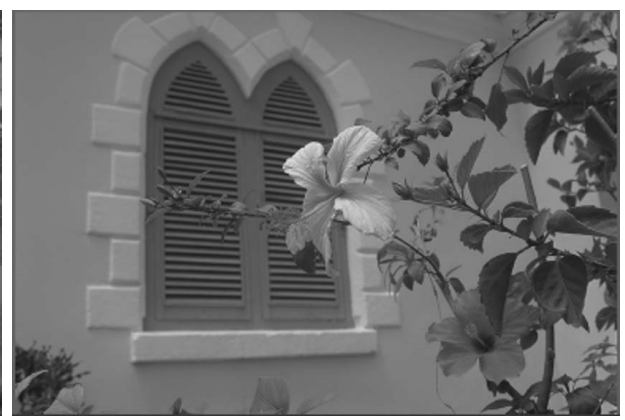

(f) NLCV induced by the optimal one with window size $40 \times 40$ : MC 20.80

Figure 4: Mean Contrast (MC) of luminance images (66). Comparisons between the luminance of original color images (top row) and the luminance of the solutions (29) of the local contrast reduction models (28) for different nonlocal covariant derivative (NLCV). 


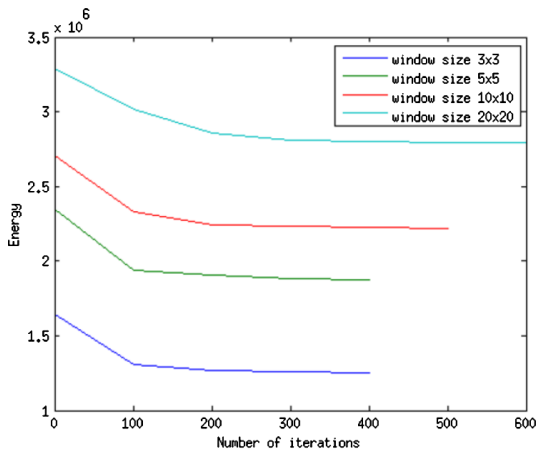

(a) Algorithm tested on Fig. 2(a) with the trivial covariant derivative

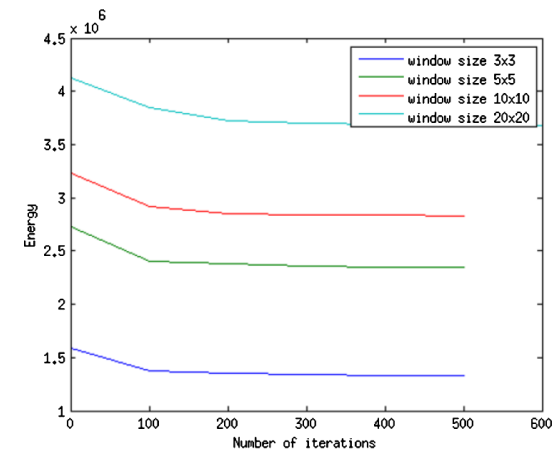

(c) Algorithm tested on Fig. 3(a) with the trivial covariant derivative

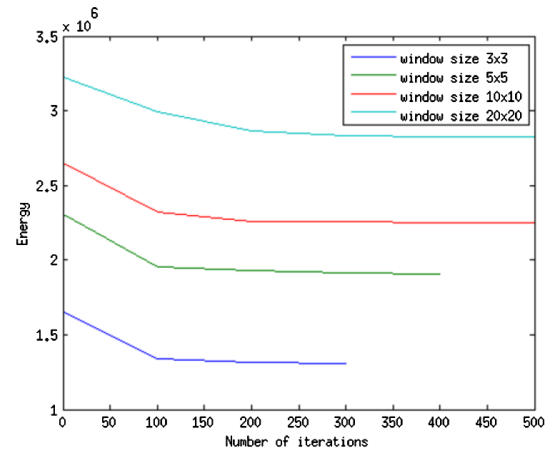

(b) Algorithm tested on Fig. 2(a) with the optimal covariant derivative

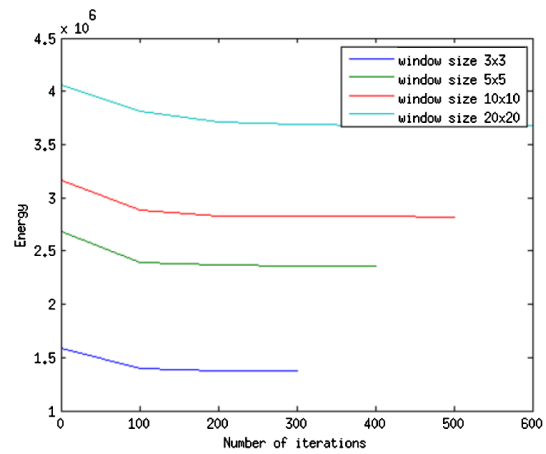

(d) Algorithm tested on Fig. 3(a) with the optimal covariant derivative

Figure 5: Plot of the energy (28) at different iterations of the algorithm (37) until convergence for different covariant derivatives and window sizes. (Color figure online)

the small details of the image for such window sizes, and the smoothing decreases with the increase of the window size.

Comparing the results obtained with the two different covariant derivatives, we observe that the colors are less saturated in the case of the optimal one (compare Fig. 7-9(b) and Fig. 7-9(c)), making the colors be closer to the ones of the original images and consequently the aspect of the original images be more preserved. This behavior was already observed in the previous Section dealing with local contrast reduction.

A more objective way to determine whether the aspect of the original image has been more preserved using the optimal covariant derivative is to 


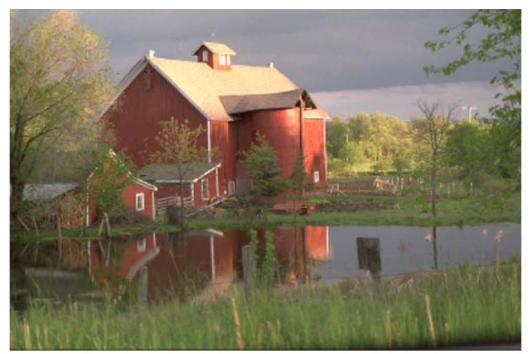

(a) Original Image $u_{0}$
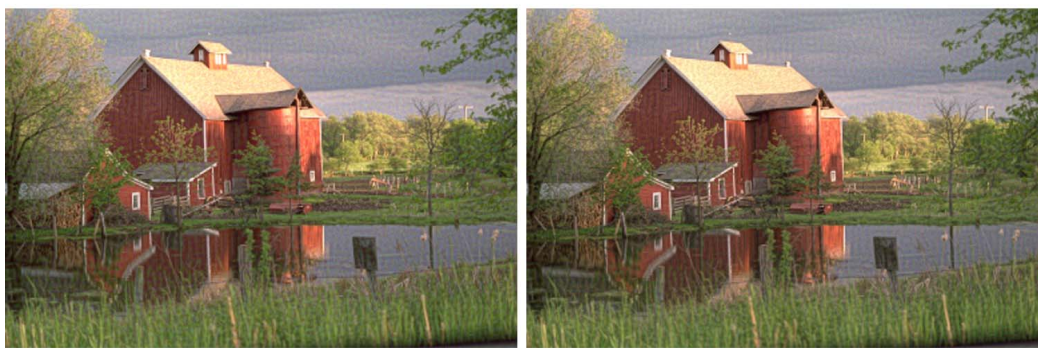

(b) NLCV induced by the trivial one with (c) NLCV induced by the optimal one window size $5 \times 5$ with window size $5 \times 5$
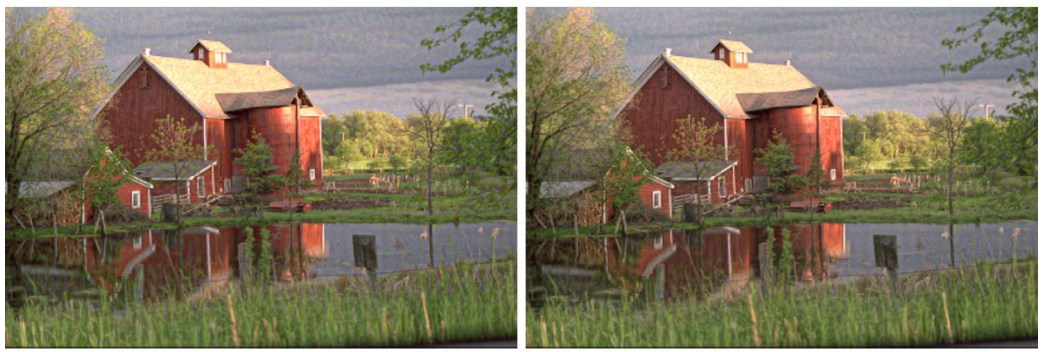

(d) NLCV induced by the trivial one with (e) NLCV induced by the optimal one window size $10 \times 10$ with window size $10 \times 10$
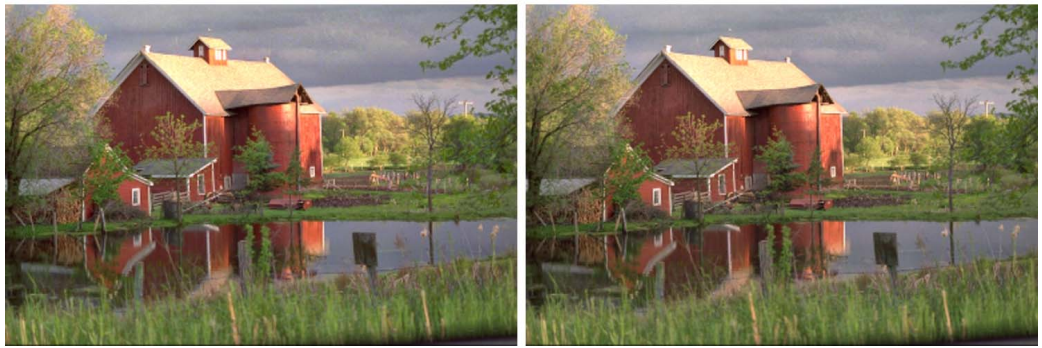

(f) NLCV induced by the trivial one with (g) NLCV induced by the optimal one window size $40 \times 40$ with window size $40 \times 40$

Figure 6: Solutions $\underline{u}$ (42) of the local contrast enhancement model (41) for different nonlocal covariant derivatives (NLCV). (Color figure online) 


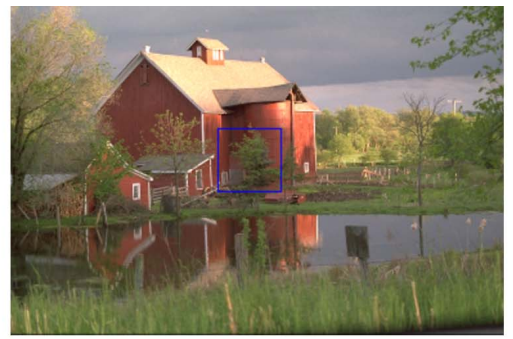

(a) Original Image $u_{0}$
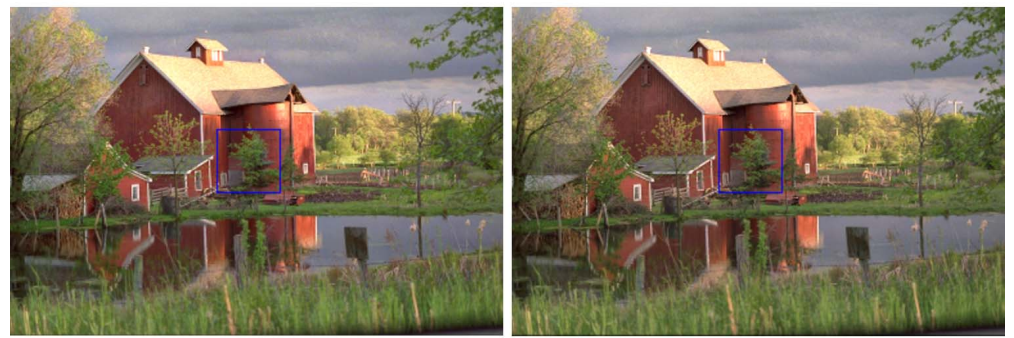

(b) NLCV induced by the trivial one with (c) NLCV induced by the optimal one window size $40 \times 40$ with window size $40 \times 40$

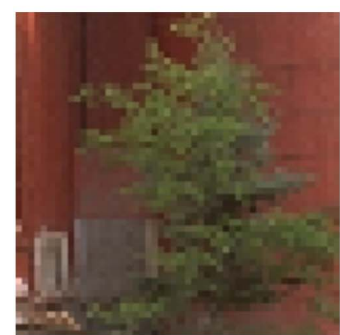

(d) Close-up of (a)

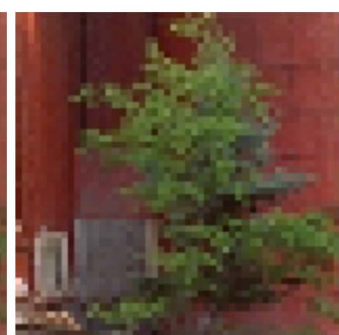

(e) Close-up of (b)

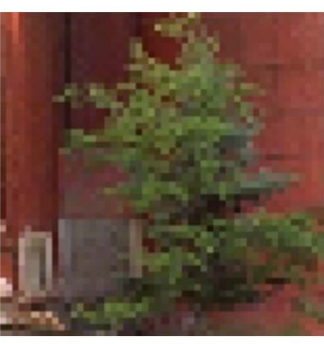

(f) Close-up of (c)
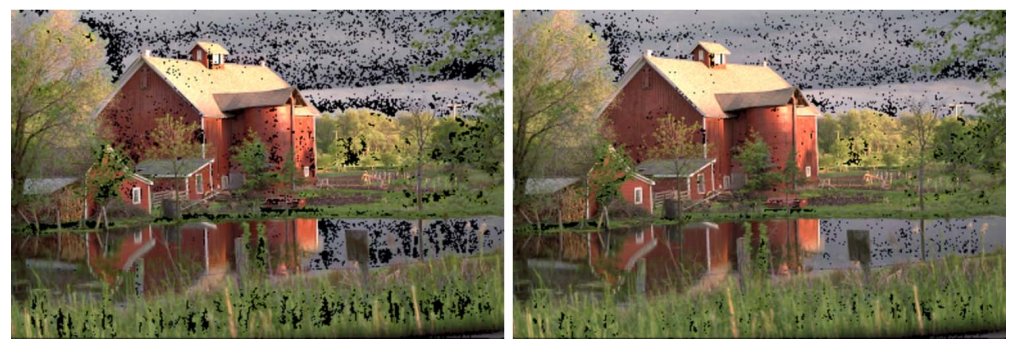

(g) $10.03 \%$ of the colors of (b) are outside (h) $4.17 \%$ of the colors of (c) are outside the convex hull of the colors of (a) the convex hull of the colors of (a)

Figure 7: $(\mathrm{b}-\mathrm{c})$ : solutions $\underline{u}(42)$ of the local contrast enhancement model (41) applied to (a) for different nonlocal covariant derivatives (NLCV). Black pixels on $(\mathrm{g}-\mathrm{h})$ show the colors of $(\mathrm{b}-\mathrm{c})$ that lie outside the convex hull of the colors of $(\mathrm{a})$. (Color figure online) 


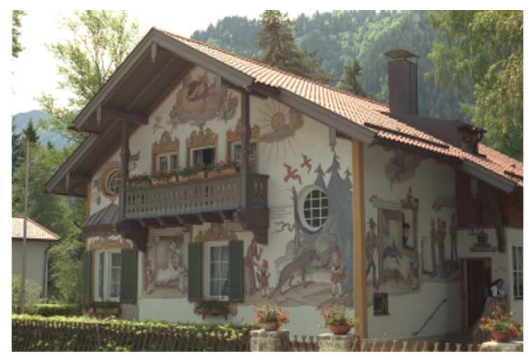

(a) Original Image $u_{0}$
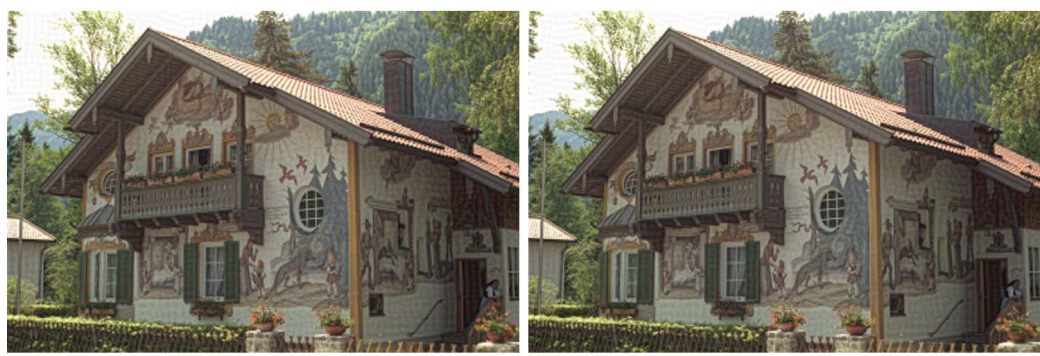

(b) NLCV induced by the trivial one with (c) NLCV induced by the optimal one window size $5 \times 5$ with window size $5 \times 5$
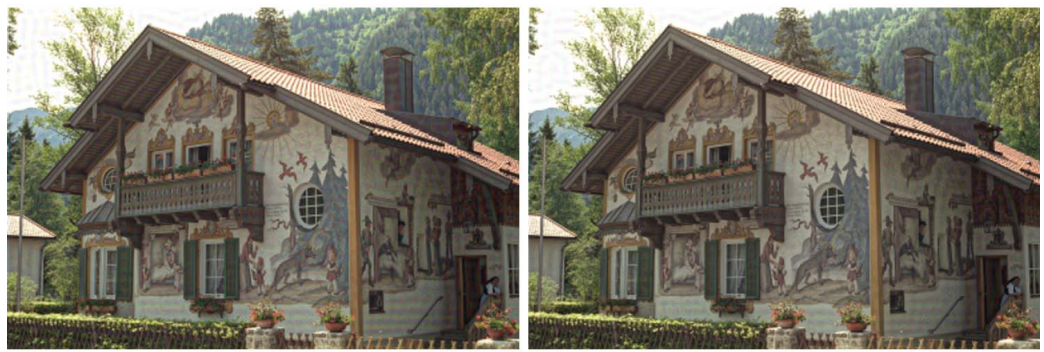

(d) NLCV induced by the trivial one with (e) NLCV induced by the optimal one window size $10 \times 10$ with window size $10 \times 10$
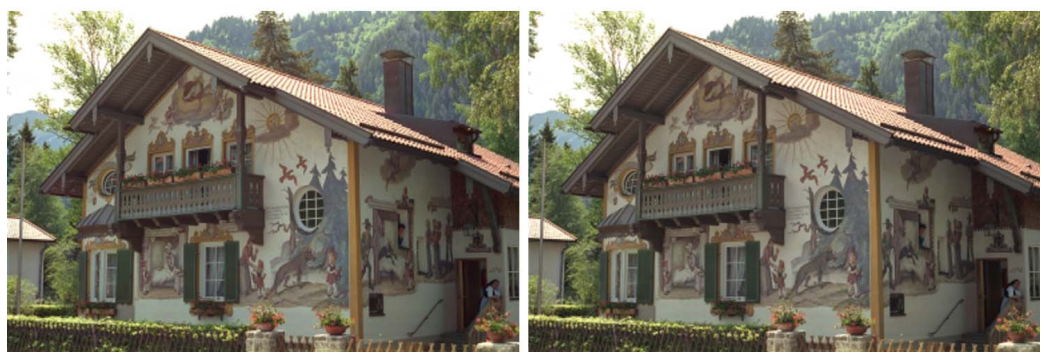

(f) NLCV induced by the trivial one with (g) NLCV induced by the optimal one window size $40 \times 40$ with window size $40 \times 40$

Figure 8: Solutions $\underline{u}$ (42) of the local contrast enhancement model (41) for different nonlocal covariant derivatives (NLCV). (Color figure online) 


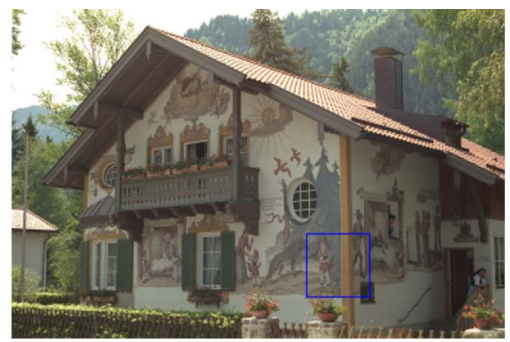

(a) Original Image $u_{0}$
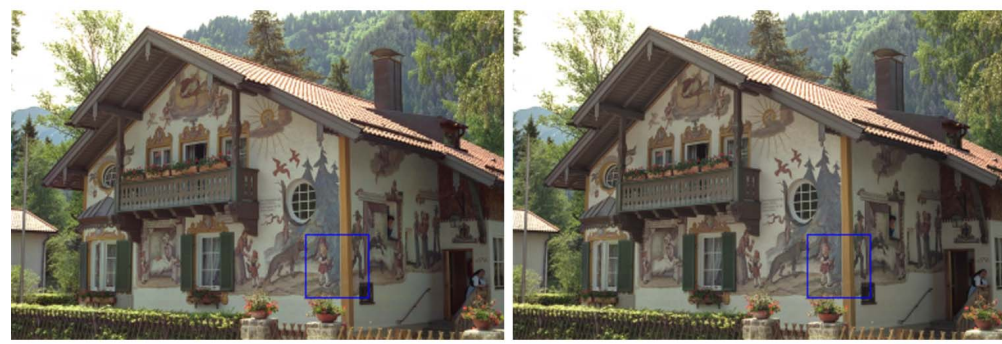

(b) NLCV induced by the trivial one with (c) NLCV induced by the optimal one window size $40 \times 40$ with window size $40 \times 40$

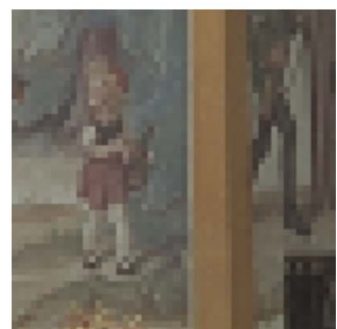

(d) Close-up of (a)

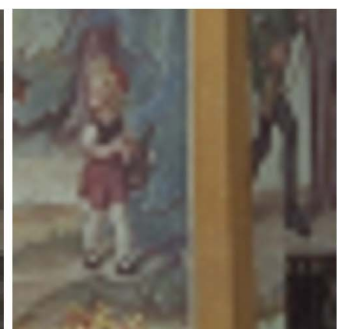

(e) Close-up of (b)

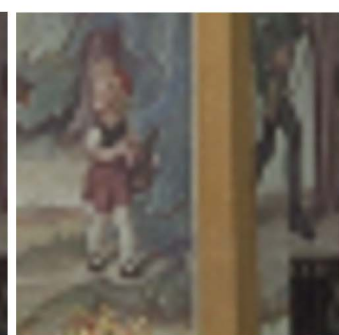

(f) Close-up of (c)
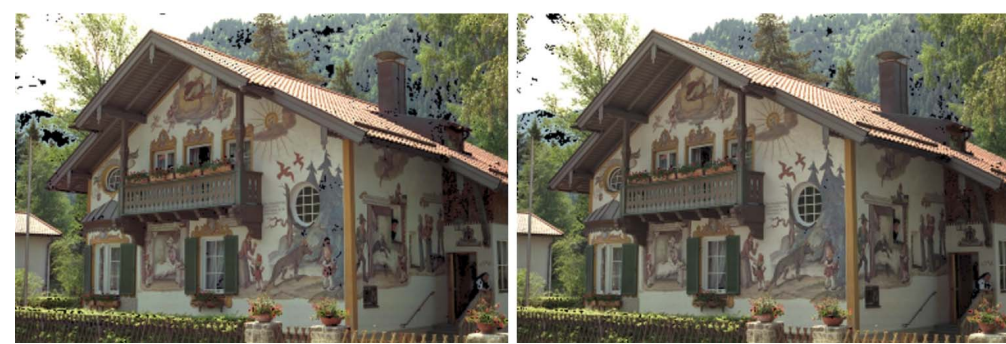

(g) $2.52 \%$ of the colors of (b) are outside (h) $1.21 \%$ of the colors of (c) are outside the convex hull of the colors of (a) the convex hull of the colors of (a)

Figure 9: (b-c): solutions $\underline{u}(42)$ of the local contrast enhancement model (41) applied to (a) for different nonlocal covariant derivatives (NLCV). Black pixels on $(\mathrm{g}-\mathrm{h})$ show the colors of $(\mathrm{b}-\mathrm{c})$ that lie outside the convex hull of the colors of (a). (Color figure online) 
compute how many colors in the contrast enhanced image (Fig. 7-9(b) and Fig. 7-9(c)) belong to the convex hull of the colors of the original image (Fig. 7-9(a)). The black pixels of the images on Fig. 7-9(g-h) represent the colors of Fig. 7-9(b-c) that lie outside the convex hulls of the corresponding original images Fig. 7-9(a), and we see that there are less black pixels on Fig. 7-9(h) than on Fig. 7-9(g), meaning that the contrast enhanced images induced by the optimal covariant derivative on Fig. 7-9(c) have more colors inside the convex hulls of the original images than the ones induced by the trivial covariant derivative on Fig. 7-9(b).

As we did in the previous Section, in order to show that the better preservation of the aspect of the original images using the optimal covariant derivative is not due to the fact that the contrast is less enhanced, we compute and compare the contrast measure (66) of the luminance of the images on Fig. 7-9(b) and Fig. 7-9(c). Results are presented in Fig. 10, and the contrast measure tells us that the model based on the optimal covariant derivative enhances equally (even a bit more) the contrast than the one based on the trivial covariant derivative.

The experiments on local contrast enhancement that we have conducted in this Section yield the same conclusion as the ones of the previous Section on local contrast reduction, i.e. using the optimal covariant derivative makes the model preserve more the aspect of the original image than the trivial covariant derivative does while enhancing (almost) equally its contrast.

Finally, we show on Fig. 11 the plots of the energy (41) at different iterations of the algorithm (56) until convergence applied to the images on Fig. 6(a) and Fig. 8(a) and tested with different window sizes and the two covariant derivatives aforementioned.

\subsection{Functional spaces modeling noise, textures and contrast on color images}

Given a color image $u_{0}$ defined on a discrete grid $\Omega$, we showed in Sect. 3 that the solutions $\underline{u}$ of both models (28) and (41) are of the form

$$
\underline{u}=u_{0}-u^{\star}
$$

where $u^{\star}$ is an element of the space $K_{1 / \lambda}$ defined by

$$
K_{1 / \lambda}:=\left\{\nabla_{w}^{N L^{*}} \eta: \eta \in \Gamma\left(p r_{1}(E)\right),\|\eta(x, y)\|_{h_{x}} \leq 1 / \lambda \forall x, y \in \Omega\right\}
$$

According to the experiments performed in Sect. 4.3-4.4 (where the vector bundle metric $h$ has been taken as the Euclidean scalar product in the $R G B$ 


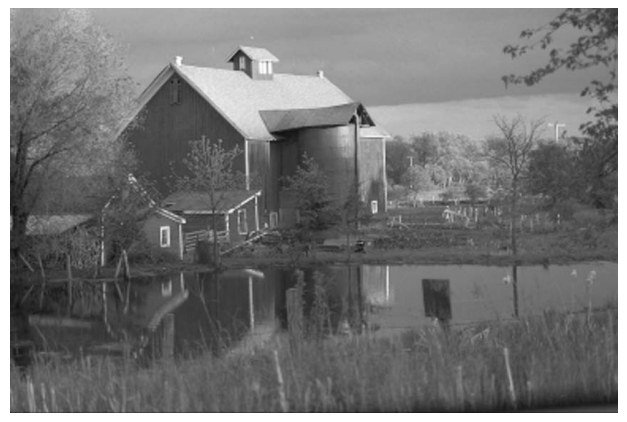

(a) Original Image: MC 20.65

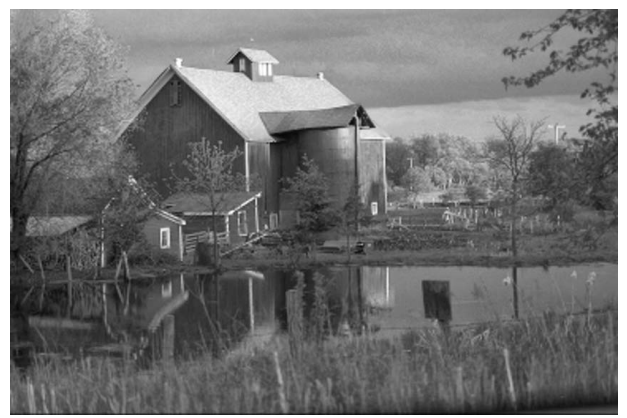

(c) NLCV induced by the trivial one with window size $40 \times 40$ : MC 25.60

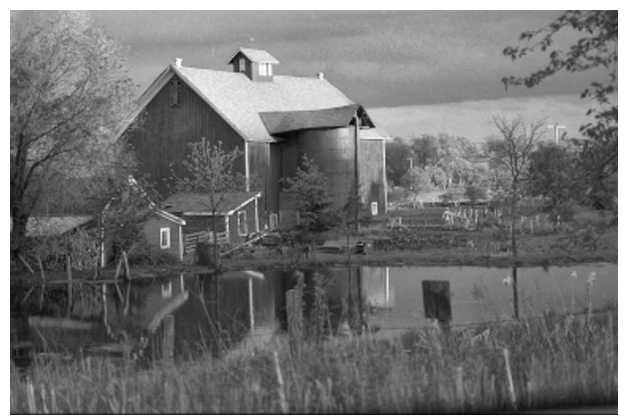

(e) NLCV induced by the optimal one with window size $40 \times 40$ : MC 25.96

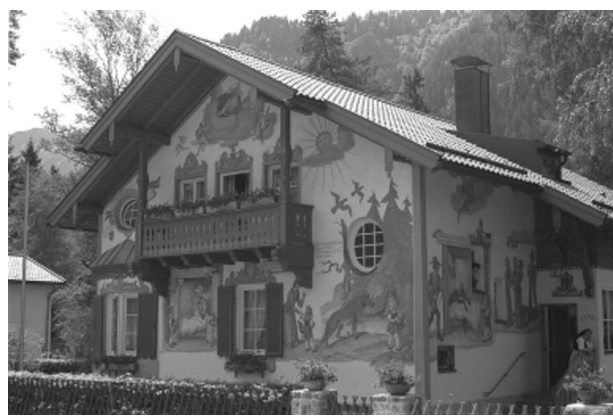

(b) Original Image: MC 29.96

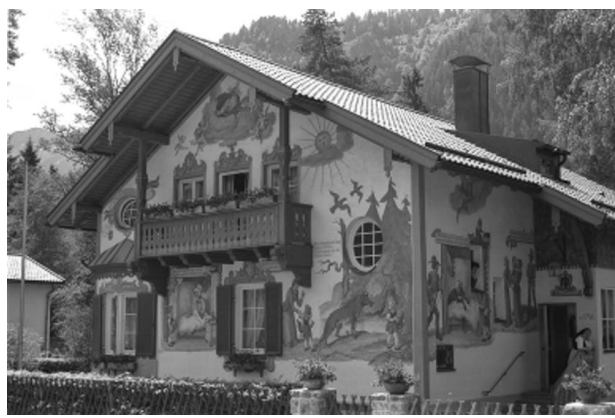

(d) NLCV induced by the trivial one with window size $40 \times 40$ : MC 35.11

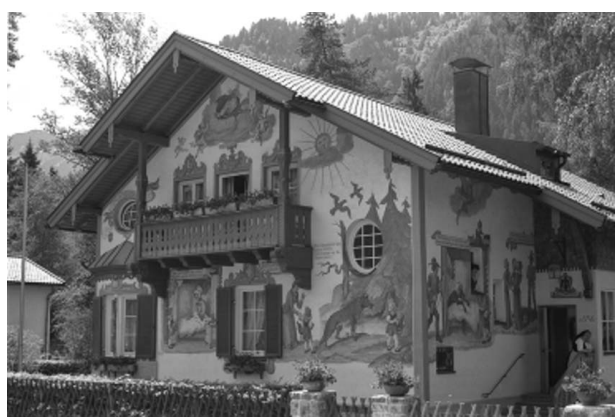

(f) NLCV induced by the optimal one with window size $40 \times 40$ : MC 35.39

Figure 10: Mean Contrast (MC) of luminance images (66). Comparisons between the luminance of original color images (top row) and the luminance of the solutions (42) of the local contrast reduction models (41) for different nonlocal covariant derivative (NLCV). 

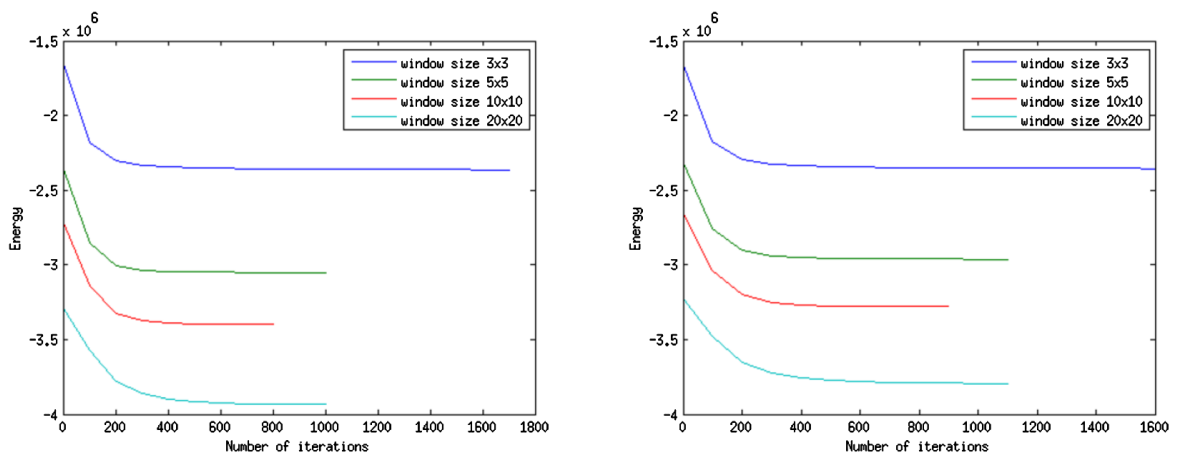

(a) Algorithm tested on Fig. 6(a) with (b) Algorithm tested on Fig. 6(a) with the trivial covariant derivative the optimal covariant derivative
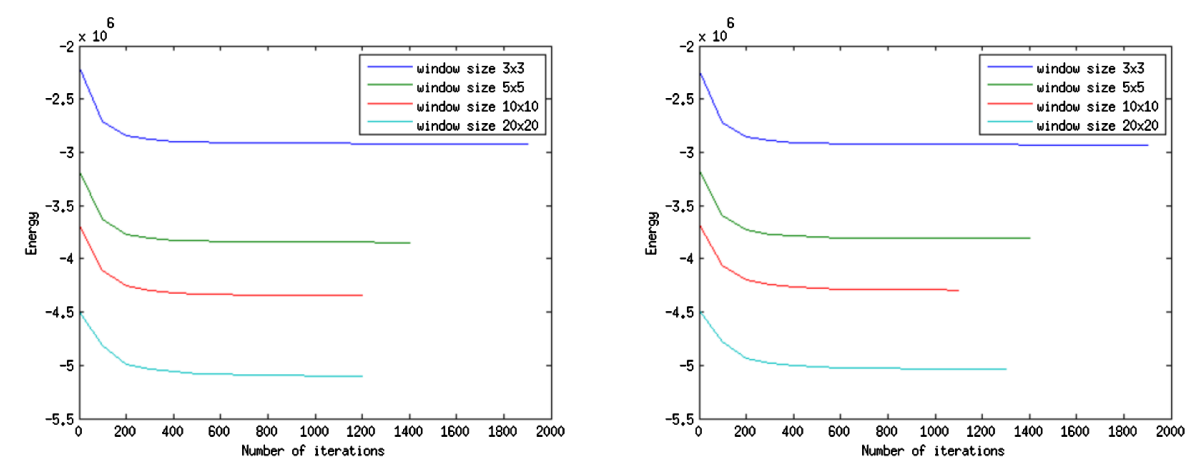

(c) Algorithm tested on Fig. 8(a) with (d) Algorithm tested on Fig. 8(a) with the trivial covariant derivative the optimal covariant derivative

Figure 11: Plot of the energy (41) at different iterations of the algorithm (56) until convergence for different covariant derivatives and window sizes. (Color figure online)

color space in each fiber, and the function $w$ is a truncated (normalized) Gaussian kernel), these variational models reduce or enhance the local contrast of the original image $u_{0}$, the parameter $\lambda$ being inversely proportional to the intensity of the reduction or enhancement of the local contrast, and the window size determines the features involved: a very small window size makes the variational models affect mainly the noise and the small details of the image like its textures and very few its contrast, whereas a larger window size makes the variational models affect mainly the contrast of the image. We can then intuit that the spaces $K_{1 / \lambda}$ (associated to the trivial and optimal covariant derivatives aforementioned), model noise, textures and contrast of color images. In what follows, we aim at justifying these observations. 
4.5.1. On the relation between large window sizes and contrast In [7], Bertalmío et al. solved the enhancement model (38) through an iterative procedure and determined properties of the model by analyzing the evolution of the initial image at each iteration. Indeed, they showed that, assuming that the kernel $w$ is flat on the whole image domain $\Omega$, i.e. $w \equiv 1 /|\Omega|$, the model (38) as well as its vectorial extension (39) preserve some structure of the initial image $u_{0}$. More precisely, they showed that the following property is satisfied

$$
u_{0}(x)=u_{0}(y) \Longrightarrow \underline{u}(x)=\underline{u}(y) \quad \forall x, y \in \Omega
$$

We intuit that this property makes the enhancement model (39) mainly affect the global structures of the image, like its contrast, and very few its local structures like noise and textures.

This property is not satisfied anymore when the kernel $w$ is not flat. By the large variance value (2000) we take in the experiments, the kernels we are considering can be seen as flat kernels on the windows they are defined. We then deduce that increasing the window size makes the Euclidean model (39) tend to satisfy the property (69), as it makes the kernel tend towards the flat kernel $1 /|\Omega|$. It explains the results on Fig. 6-8, and especially why, for the largest window size we consider in our experiments, i.e. $40 \times 40$, we mainly observe contrast enhancement and few noise increase (see Fig. 6-8(f)).

The property (69) is not necessarily satisfied in the more general case of the model (41) due to the presence of the parallel transport map $\tau_{x, y}^{-1}$ in the expression of the non-local total variation (22). However, the experiments we conducted with the optimal covariant derivative showed the same behavior as in the Euclidean case: large window sizes make the model mainly affect the contrast and very few the local components as textures/noise. We intuit that this is related to the fact that the optimal covariant derivative is compatible with respect to the Euclidean norm in $R G B$, which makes the transport parallel map preserve the norm, leading to the following upper and lower bounds for the term $\left\|\tau_{(x, y)}^{-1} u(y)-u(x)\right\|$ in the expression $(22)$

$$
|\|u(x)\|-\|u(y)\|| \leq\left\|\tau_{(x, y)}^{-1} u(y)-u(x)\right\| \leq\|u(x)\|+\|u(y)\|
$$

which are the same bounds as in the Euclidean case, since we have

$$
|\|u(x)\|-\|u(y)\|| \leq\|u(y)-u(x)\| \leq\|u(x)\|+\|u(y)\|
$$

Finally, the analysis performed in [7] can be adapted to the regularization model (25) and its vectorial extension (26) in a straightforward way as the 
proposed enhancement and regularization models only differ by the sign of the non-local total variation, and it can be easily shown that the property (69) also holds in the regularization models. As in the enhancement case, we observe that, if the window size is large enough, then the regularization mainly affect the contrast, whereas the textures tend to be preserved (see Fig. $2-3(\mathrm{f}-\mathrm{g})$ where a window size $40 \times 40$ has be taken).

\subsubsection{On the relation between small window sizes and noise/tex-}

tures Dealing with small window sizes, we observed the opposite behavior as the one observed with large window sizes, i.e. the local structures like textures and noise are much more affected than the contrast. This behavior is related to the fact that the solutions of the regularizations and enhancement models are of the form (67). Indeed, in the scalar case and when the covariant derivative is the trivial one, reducing the window size makes the space (68) tends towards the space

$$
\left\{\nabla^{*} \eta: \eta \in C^{\infty}\left(\Omega ; \mathbb{R}^{2}\right),|\eta(x)| \leq 1 / \lambda \forall x \in \Omega\right\},
$$

where $\nabla^{*}$ is the adjoint of the Euclidean gradient operator, which is a subspace of the space of oscillating patterns introduced by Meyer [29], that includes textures and noise. It follows from (67) that the regularization and enhancement models consist in removing some oscillating patterns from the original image $u_{0}$. Dealing with the regularization models, the oscillating patterns removed from the original image make the models remove the noise and smooth the textures (see Fig. 2-3(b-c)), whereas the ones removed in the case of enhancement models make these latter enhance the noise and textures (see Fig. 6-8(b-c)).

Finally, it is worth noting, that reducing the window size makes the regularization model tend towards the ROF denoising model [33] which has the tendency of reducing (slightly) the contrast, which might explain why our models affect slightly the contrast for small window sizes.

4.5.3. On the residual term On Fig. 12-13, we show the results of the differences between the solutions of both variational models (28) and (41) applied to the same original image $u_{0}$ and the original image itself, i.e. we show the element $u^{\star}$ in (67), which is given by

$$
u^{\star}=\underset{v \in K_{1 / \lambda}}{\arg \min }\left\|u_{0}-v\right\|_{L^{2}}^{2},
$$

in the local contrast reduction model (see Fig. 12), and by 


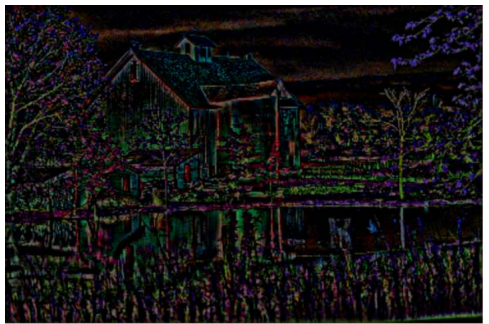

(a) NLCV induced by the trivial one with window size $10 \times 10$ : Negative part

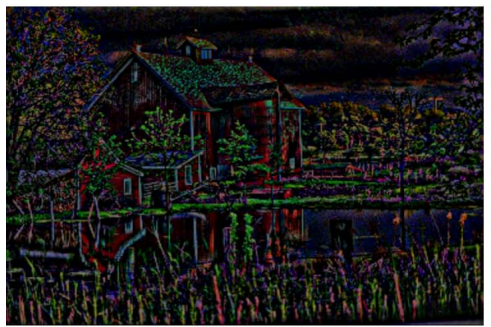

(c) NLCV induced by the trivial one with window size $10 \times 10$ : Positive part

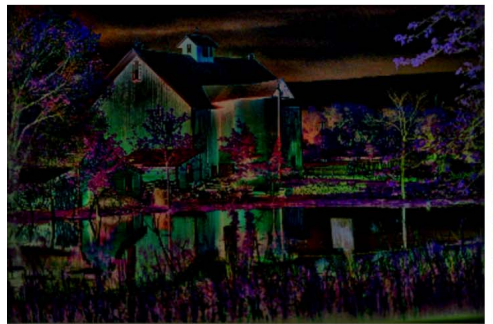

(e) NLCV induced by the trivial one with ( window size $40 \times 40$ : Negative part

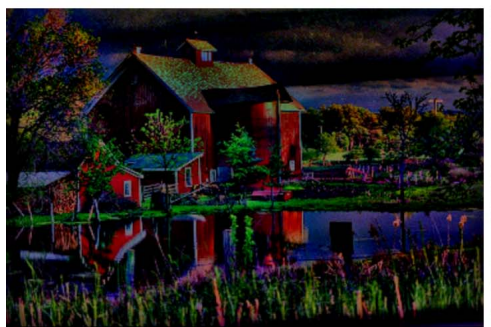

(g) NLCV induced by the trivial one with window size $40 \times 40$ : Positive part

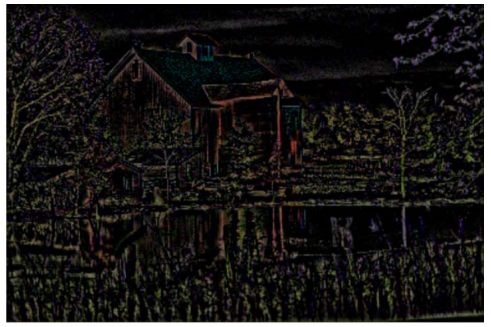

(b) NLCV induced by the optimal one with window size $10 \times 10$ : Negative part

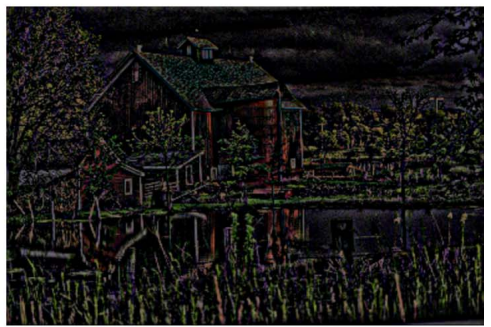

(d) NLCV induced by the optimal one with window size $10 \times 10$ : Positive part

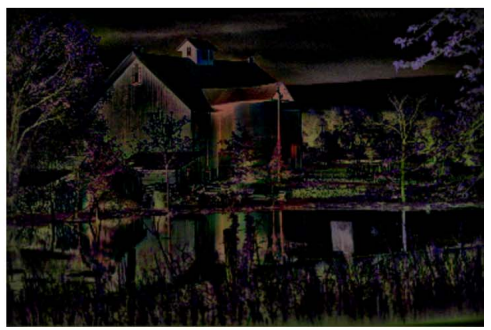

LCV induced by the optimal one with window size $40 \times 40$ : Negative part

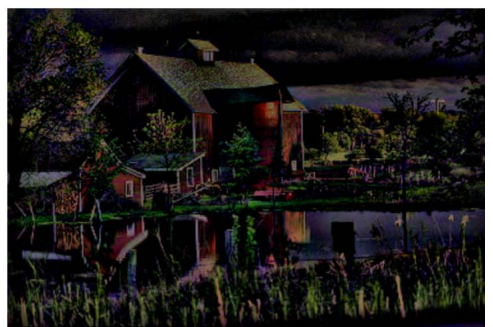

(h) NLCV induced by the optimal one with window size $40 \times 40$ : Positive part

Figure 12: Difference $u^{\star}=u_{0}-\underline{u}$ (71) for the local contrast reduction model with different nonlocal covariant derivatives (NLCV) applied to $u_{0}$ on Fig. 2(a). Values multiplied by 10 for visualization purposes. (Color figure online) 

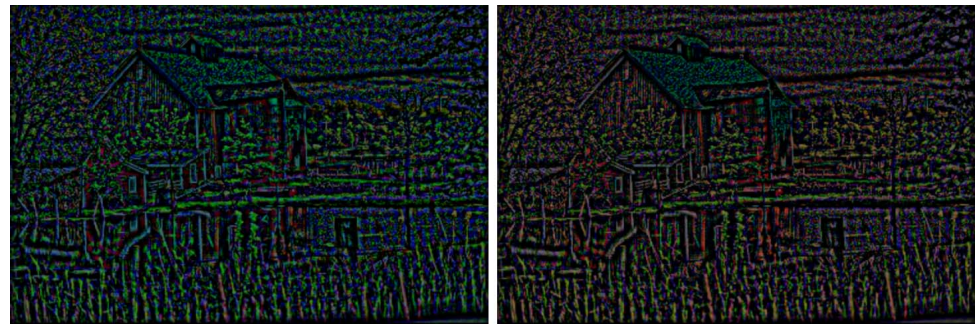

(a) NLCV induced by the trivial one with (b) NLCV induced by the optimal one window size $10 \times 10$ : Negative part with window size $10 \times 10$ : Negative part
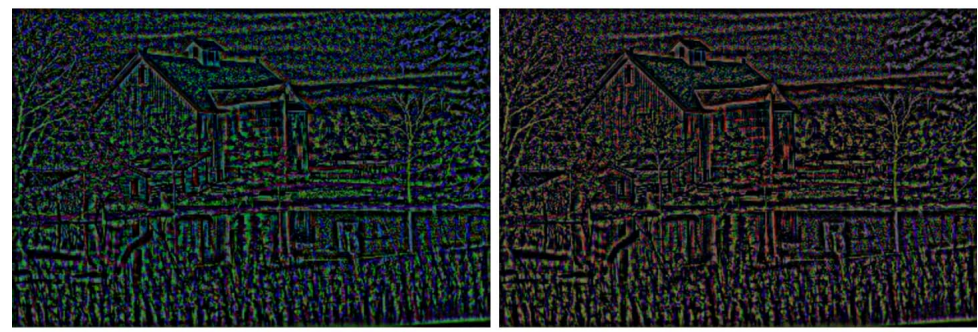

(c) NLCV induced by the trivial one with (d) NLCV induced by the optimal one window size $10 \times 10$ : Positive part with window size $10 \times 10$ : Positive part
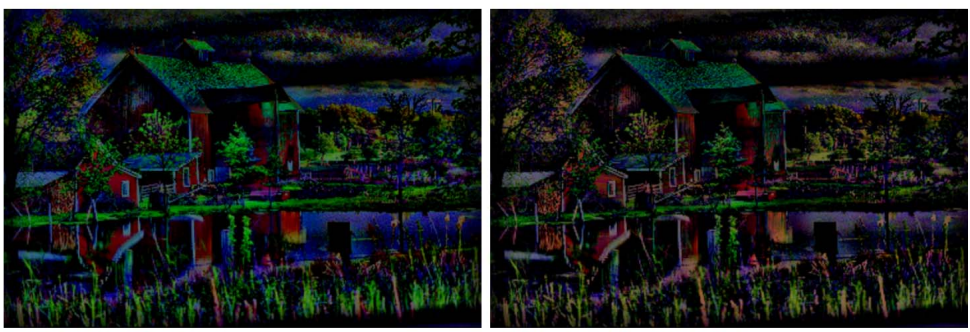

(e) NLCV induced by the trivial one with (f) NLCV induced by the optimal one window size $40 \times 40$ : Negative part with window size $40 \times 40$ : Negative part
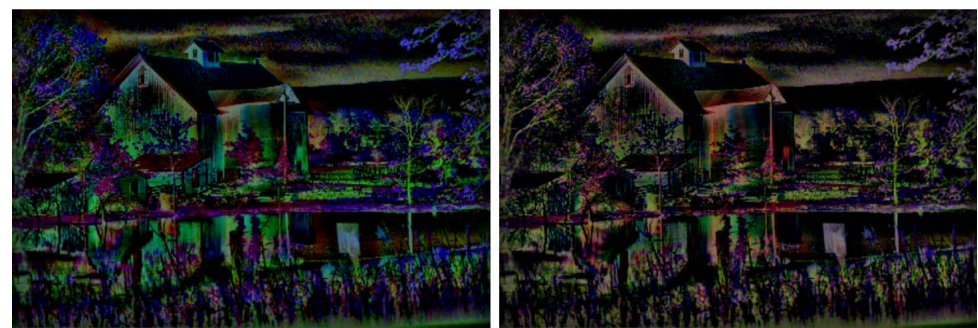

(g) NLCV induced by the trivial one with (h) NLCV induced by the optimal one window size $40 \times 40$ : Positive part with window size $40 \times 40$ : Positive part

Figure 13: Difference $u^{\star}=u_{0}-\underline{u}$ (72) for the local contrast enhancement model with different nonlocal covariant derivatives (NLCV) applied to $u_{0}$ on Fig. 2(a). Values multiplied by 10 for visualization purposes. (Color figure online) 


$$
u^{\star}=\underset{v \in K_{1 / \lambda}}{\arg \max }\left\|u_{0}-v\right\|_{L^{2}}^{2}
$$

in the local contrast enhancement model (see Fig. 13).

We test both models with the two covariant derivatives aforementioned and two different window sizes, i.e. $10 \times 10$ and $40 \times 40$. Moreover, we use the same parameter value $\lambda=0.1$ and stopping criteria (65).

Through the observation of the positive and negative parts of $u^{\star}$ and the comparisons between the two different window sizes, we deduce a region-wise oscillating behavior of $u^{\star}$ around the value 0 , that tends to get pixel-wise when reducing the window size. We also observe color oscillations of smaller saturation in the case of the optimal covariant derivative, which explains why this covariant derivative makes the regularization and enhancement models preserve more the colors of the original image. Finally, a comparison between the results on Fig. 12 and Fig. 13 reveals big similarities in the case of the window size $40 \times 40$ between the positive part of the local contrast reduction model and the negative part of the local contrast enhancement model and vice-versa, in the sense that the elements $u^{\star}$ are close to be opposite. This result is consistent with the expressions of $u^{\star}$ in (71) and (72) and the fact that $K_{1 / \lambda}$ is the image of a ball through a linear map. However, the similarities are less obvious for the small window size $10 \times 10$.

\section{Conclusion and further work}

We have introduced the notion of nonlocal covariant derivative on a vector bundle from which we have derived the concept of nonlocal total variation for sections of a vector bundle. Then we have provided an application of these new mathematical tools to image processing by extending existing variational models devoted to local contrast reduction/enhancement from the Euclidean space to a vector bundle. For a well-chosen nonlocal covariant derivative, we have shown that the models preserve more the aspect of the original image than the existing Euclidean models do, while reducing/enhancing equally its contrast. As contrast reduction/enhancement is deeply related with human vision and covariant differentiation models some properties of the human visual system too, we are investigating the relations between the proposed mathematical model and human vision.

The proposed algorithms are quite slow, which make them be unpractical in their current state. It is mainly due to the fact that the nonlocal covariant derivative between two points is the solution of an ODE, whose explicit solution is unknown, and has then to be solved through a numerical scheme. This computational issue forces us to deal with small window sizes and it makes the models act on noise and textures while modifying the im- 
age contrast, which can be undesirable in some applications where contrast reduction/enhancement is useful. Current work is then devoted to speeding up the algorithm in order to make it applicable on large window sizes.

\section{Acknowledgements}

The authors thank the anonymous reviewers for helpful comments.

\section{References}

[1] S. P. Awate, R. T. Whitaker (2006). Unsupervised, informationtheoretic, adaptive image filtering for image restoration. IEEE Trans. Pattern Anal. Mach. Intell. 28(3) 364-376.

[2] T. Batard (2011). Heat equations on vector bundles - application to color image regularization. J. Math. Imaging Vis. 41(1-2) 59-85. MR2824148

[3] T. Batard, N. Sochen (2014). A class of generalized Laplacians devoted to multi-channels image processing. J. Math. Imaging Vis. 48(3) 517543. MR3171428

[4] T. Batard, M. Bertalmío (2014). On covariant derivatives and their applications to image regularization. SIAM J. Imaging Sci. 7(4) 23932422. MR3280098

[5] T. Batard, M. Bertalmío (2015). Duality principle for image regularization and perceptual color correction models. Lecture Notes in Computer Science, Springer 9087 449-460.

[6] M. Bertalmío (2014). Image Processing for Cinema. Chapman \& Hall/CRC.

[7] M. Bertalmío, V. Caselles, E. Provenzi, A. Rizzi (2007). Perceptual color correction through variational techniques. IEEE Trans. Im. Processing 16(4) 1058-1072. MR2463909

[8] M. Bertalmío, V. Caselles, E. Provenzi (2009). Issues about Retinex theory and contrast enhancement. Int. J. Computer Vis. 83(1) 101119 .

[9] M. Bertalmío, J. D. Cowan (2009). Implementing the Retinex algorithm with Wilson-Cowan equations. J. Physiology 103(1-2) 69-72.

[10] K. Bredies, K. Kunish, T. Pock (2010). Total generalized variation. SIAM J. Imaging Sci. 3(3) 492-526. MR2736018 
[11] X. Bresson, T. F. Chan (2008). Fast dual minimization of the vectorial total variation norm and applications to color image processing. Inverse Probl. Imaging 2(4) 455-484. MR2558277

[12] A. Buades, B. Coll, J.-M. Morel (2005). A non-local algorithm for image denoising. Proceedings of CVPR (2) 60-65.

[13] A. Chambolle (2004). An algorithm for total variation minimization and applications. J. Math. Imaging Vis. 20(1-2) 89-97. MR2049783

[14] A. Chambolle, T. Pock (2011). A first-order primal-dual algorithm for convex problems with applications to imaging. J. Math. Imaging Vis. 40(1) 120-145. MR2782122

[15] S. Ferradans, M. Bertalmío, E. Provenzi, V. Caselles (2011). An analysis of visual adaptation and contrast perception for tone mapping. IEEE Trans. Pattern Anal. Mach. Intell. 33(10) 2002-2012.

[16] J. H. Fitschen, M. Nikolova, F. Pierre, G. Steidl (2015). A variational model for color assignment. Lecture Notes in Computer Science, Springer 9087 437-448.

[17] E. Franken, R. Duits (2009). Crossing-preserving coherence-enhancing diffusion on invertible orientation scores. Int. J. Computer Vis. 85(3) $253-278$.

[18] A. Galdran, J. Vazquez-Corral, D. Pardo, M. Bertalmío (2015). Enhanced variational image dehazing. SIAM J. Imaging Sci. 8(3) 15191546. MR3373630

[19] T. Georgiev (2005). Relighting, Retinex theory, and perceived gradients. Proc. Int. Conf. Computer Vis./Computer Graphics Collaboration Techniques and Applications MIRAGE.

[20] G. Gilboa, S. Osher (2008). Nonlocal operators with applications to image processing. Multiscale Model. Simul. 7(3) 1005-1028. MR2480109

[21] B. Goldluecke, E. Strekalovskiy, D. Cremers (2012). The natural vectorial total variation which arises from geometric measure theory. SIAM J. Imaging Sci. 5(2) 537-563. MR2971172

[22] B. He, X. Yuan (2012). Convergence analysis of primal-dual algorithms for a saddle-point problem: from contraction perspective. SIAM J. Imaging Sci. 5(1) 119-149. MR2902659

[23] Y. Jin, J. Jost, G. Wang (2014). A new nonlocal $H^{1}$ model for image denoising. J. Math. Imaging Vis. 48(1) 93-105. MR3147612 
[24] J. Jost (2008). Riemannian Geometry and Geometric Analysis. Springer, Berlin. MR2431897

[25] S. Kindermann, S. Osher, P. W. Jones (2005). Deblurring and denoising of images by nonlocal functionals. Multiscale Model. Simul. 4(4) 10911115. MR2203846

[26] E. Land, J. McCann (1971). Lightness and Retinex theory. J. Optical Society of America 61(1) 1-7.

[27] H. B. Lawson, M.-L. Michelson (1989). Spin Geometry. Princeton University Press. MR1031992

[28] J. M. Lee (1997). Riemannian Manifolds: An Introduction to Curvature. Springer. MR1468735

[29] Y. Meyer (2001). Oscillating patterns in image processing and in some nonlinear evolution equations. The Fifteenth Dean Jacqueline B. Lewis Memorial Lectures. American Mathematical Society. MR1852741

[30] R. Palma-Amestoy, E. Provenzi, M. Bertalmío, V. Caselles (2009). A perceptually inspired variational framework for color enhancement. IEEE Trans. Pattern Anal. Mach. Intell. 31(3) 458-474.

[31] M. Pedersen, J. Hardeberg (2011). Full-reference image quality metrics: classification and evaluation. Found. Trends Comp. Graphics and Vision 7 1-80.

[32] R. T. Rockafellar (1970). Convex Analysis. Princeton University Press. MR0274683

[33] L. I. Rudin, S. Osher, E. Fatemi (1992). Nonlinear total variation based noise removal algorithms. Physica D 60 259-268. MR3363401

[34] G. Sapiro, V. Caselles (1997). Histogram modification via differential equations. J. Differential Equations 135(2) 238-268. MR1441271

[35] N. Sochen, R. Kimmel, R. Malladi (1998). A general framework for low level vision. IEEE Trans. Im. Processing 7(3) 310-318. MR1669540

[36] J. F. Tolan (1979). A duality principle for non-convex optimisation and the calculus of variations. Archiv. Rational Mech. Analysis 71(1) 4161. MR0522706

[37] Z. Wang, A. Bovik (2002). A universal image quality index. IEEE Signal Process. Letters 9(3) 81-84. 
[38] D. Zosso, G. Tran, S. Osher (2015). Non-local Retinex - A unifying framework and beyond. SIAM J. Imaging Sci. 8(2) 787-826. MR3328139

[39] J. von Kries (1902). Chromatic Adaptation [Translation: D. MacAdam, "Sources of Color Science", MIT press, 1970].

[40] J. Weickert (1999). Coherence-enhancing diffusion of colour images. Im. Vis. Computing 17 201-212.

[41] S. W. Zamir, J. Vazquez-Corral, M. Bertalmío (2014). Gamut mapping in cinematography through perceptually-based contrast modification. IEEE J. Sel. Topics Signal Processing 8(3) 490-503.

[42] W. Zhu, T. Chan (2012). Image denoising using mean curvature of image surface. SIAM J. Imaging Sci. 5(1) 1-32. MR2902655

ThOMAS BATARD

Department of Information and Communications Technologies

UNIVERSiTy POMPEU FABRA

BARCELONA

SPAIN

E-mail address: thomas.batard@upf .edu

Marcelo Bertalmío

Department of Information and Communications Technologies

University Pompeu FABRA

BARCELONA

SPAIN

E-mail address: marcelo.bertalmio@upf .edu

RECEIVED 24 JuLy 2015 